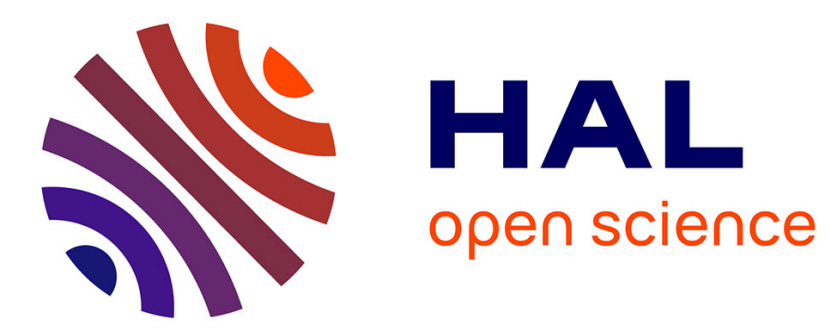

\title{
Grounding rules for (relevant) implication
}

Francesca Poggiolesi

\section{To cite this version:}

Francesca Poggiolesi. Grounding rules for (relevant) implication. Journal of Applied Non-Classical Logics, 2020, pp.26-55. 10.1080/11663081.2020.1850048 . hal-02953412

\section{HAL Id: hal-02953412 \\ https://hal.science/hal-02953412}

Submitted on 30 Sep 2020

HAL is a multi-disciplinary open access archive for the deposit and dissemination of scientific research documents, whether they are published or not. The documents may come from teaching and research institutions in France or abroad, or from public or private research centers.
L'archive ouverte pluridisciplinaire HAL, est destinée au dépôt et à la diffusion de documents scientifiques de niveau recherche, publiés ou non, émanant des établissements d'enseignement et de recherche français ou étrangers, des laboratoires publics ou privés. 
Grounding rules for (relevant) implication

Francesca Poggiolesi

Université Paris 1 Panthéon-Sorbonne, CNRS, IHPST UMR8590,

75006, Paris

France

poggiolesi@gmail.com

https://sites.google.com/site/poggiolesi/ 


\title{
Grounding rules for (relevant) implica- tion
}

\begin{abstract}
In Poggiolesi (2020a) a definition of the notion of complete and immediate formal grounding in the background of a relevant framework has been introduced; this definition generates some intuitively acceptable grounding principles for relevant implication. In the present paper our aim is to construct a logic for the notion of complete and immediate formal grounding in a relevant framework based on that definition. Our logic will have the form of a calculus of natural deduction and will formalize the relation of grounding both as a meta-linguistic relation and as a connective. The calculus will contain grounding rules for relevant implication and will be proved to be sound and complete with respect to the original definition. Finally we will prove the deduction theorem at the grounding level, i.e. we will show that grounding formalized as a metalinguistic relation is equivalent to grounding formalized as a connective.
\end{abstract}

\section{Introduction}

In the last ten years there has been a growing interest in the concept of grounding which is usually taken to be a relation amongst truths (or facts) that is non-causal and explanatory in nature. Grounding may be conveyed by the expression because although not every use of 'because' corresponds to the relation of grounding. Typical examples of grounding sentences are:

1. the glass is fragile because it has a certain molecular structure,

2. John is a bachelor because he is a man and he is unmarried,

3. the ball is red and round because the ball is red and the ball is round.

In each of these sentences the antecedent, i.e. what comes after the because ("the glass has a certain molecular structure," "John is a man and he is unmarried" and "the ball is red and the ball is round"), determines or explains the consequent, i.e. what comes before the because ("the glass is fragile," "John is a bachelor," "the ball is red and round" respectively). In other terms, in each of the sentences listed above, the antecedent constitutes a reason for or a ground of the consequent.

The literature often distinguishes between different types of grounding (e.g. see Fine (2012a)). Correia (2014) for example distinguishes between metaphysical grounding (an example of metaphysical grounding is sentence number 1), conceptual grounding (an example of conceptual grounding is sentence number 
2) and formal ${ }^{1}$ grounding (an example of formal grounding is sentence number 3). The relation between these different types of grounding is currently a topic of debate (e.g. see McSweeney (2020); Smithson (2019)). Without wishing to enter into the debate, in this paper we only focus on the notion of formal grounding that we take to be a non-causal explanation of sentences in virtue of their logical connectives.

Whatever type of grounding one is willing to take into account, other distinctions might be made. Here we focus on two, namely the distinction between complete and partial grounding, as well as the distinction between immediate and mediate grounding. The multiset ${ }^{2}$ of all, and only, those truths each of which contributes to ground a truth $A$ is a complete ground of $A .^{3}$ On the other hand, each of the truths that compose the complete ground of $A$, as well as each strict sub-multiset of them, is said to be a partial ground of $A$. As for the distinction immediate and mediate, if we could describe it in prooftheoretical terms, we would say that immediate grounding corresponds to a single (irreflexive) grounding(proof)-step, while mediate grounding corresponds to a sequence of several steps of immediate grounding. In other words, while immediate grounding is a relation that does not seem to be reducible further, mediate grounding is definable as the transitive closure of immediate grounding.

There are several studies dedicated to the formalization of the notion of grounding (e.g. see Correia (2010); Fine (2012b)). In these different studies, grounding is taken to be a primitive notion that is captured either via a predicate (e.g. see Korbmacher (2017)), or via an operator (e.g. see Correia (2014); Schnieder (2011)). The main concern is to provide grounding axioms or rules for the classical connectives of conjunction, disjunction and negation: little attention has been dedicated so far beyond classical logic, i.e. to non-classical logics, and beyond these connectives, i.e. to implication.

An alternative approach is developed by Poggiolesi (2016b, 2018, 2020a). First of all, Poggiolesi focuses on the notion of formal grounding and does not take it to be primitive; indeed, she proposes a definition of the notion of complete and immediate formal grounding in terms of the notions of derivability and g-complexity. One advantage of this approach lies in its flexibility: since formal grounding is relative to the notion of derivability and g-complexity, formal grounding is also relative to the logic in which derivability and g-complexity are defined. Hence, by considering classical derivability, and a notion of gcomplexity suited for the classical connectives, Poggiolesi (2016b) proposes a definition of the notion of complete and immediate formal grounding adequate for a classical context.

More recently, Poggiolesi (2020a) has developed a ground-theoretic study of the connective of implication that is supposed to reflect some natural intuitions

\footnotetext{
${ }^{1}$ Although Correia (2014) uses the term logical grounding, here we follow Poggiolesi (2016b) and call it 'formal.'

${ }^{2} \mathrm{~A}$ multiset is a set where the number of occurrences of the same formula counts.

${ }^{3}$ As explained in detail in Poggiolesi (2016b,a), the distinctions complete-partial and fullpartial, although similar, are not the same. A study of the relations between these distinctions can be found in Poggiolesi (2020b).
} 
about the grounds of conditionals in natural language. The starting point of this study is the consideration of some ordinary language conditionals such as:

4. if the glass is thrown, then it falls,

5. if the ball is pushed, it will roll,

6. if it rains, it is not the case that the road will not be wet.

What are the reasons for the truth of these sentences which all have the form "if $A$, then $B$ "? For the first conditional the answer seems to be the law of gravity: it is because there is the law of gravity that if the glass is thrown, then it falls. For the second conditional, the ground seems to be "the ball is a sphere:" it is because the ball is a sphere that if it is pushed, then it rolls. And for the third and last condition the answer seems to be "if it rains, the road will be wet:" it is because if it rains, then the road is wet, that if it rains, it is not the case that the road will not be wet.

These examples call for important remarks.

- First of all, they only apply to indicative conditionals where there is a connection between antecedent and consequent. This connection is very important since it is precisely what is grounded.

- Secondly, they all show the same intuitive pattern - let us call it pattern*: the ground of a conditional of the form "if $A$, then $B$ " is a sentence $C$ such that from $A$ and $C, B$ follows. ${ }^{4}$ The variety of these examples -4 and 5 are not formal, while 6 is - suggest that pattern* is a key feature of the grounding analysis of conditionals across grounding types. That said, Poggiolesi (2020a) only focuses on formal grounding as does this paper.

In Poggiolesi (2020a), the author aims at making some first steps towards the formalization of these remarks. To that end, she focuses on the relevant logic $\mathbf{R}$ where implications formalize indicative conditionals displaying a connection between antecedents and consequents: a relevant implication is indeed characterized by the fact that the antecedent is always relevant for the consequent. Formally, this feature is conveyed by means of the variable sharing principle. The variable sharing principle says that no formula of the form $A \rightarrow B$ can be proven in the relevant logic $\mathbf{R}$ if $\mathrm{A}$ and $\mathrm{B}$ do not have at least one propositional variable in common. Hence, in $\mathbf{R}$ a conditional of the form "if $2+2=4$, then the moon is yellow" is false, while a conditional of the form "if the ball is pushed, then it will roll" is true. The reason for this truth does not lie in the truth-values of its components but in the link between antecedent and consequent. And this seems to match the connection between antecedent and consequent noted in the grounding examples above.

Poggiolesi then puts forward a definition of the notion of complete and immediate formal grounding which is appropriate for the relevant framework: she

\footnotetext{
${ }^{4}$ Where following from is to be used in Anderson and Belnap (1975)'s sense: $B$ follows from $A, C$ if there is a deduction of $B$ from $A, C$ which actually uses $A, C$ (and $A, C$ alone).
} 
obtains it by adapting the key notions of derivability and g-complexity to the relevant notions. This definition not only generates some intuitively acceptable grounding principles for the usual connectives of negation, conjunction and disjunction, but also and most importantly for relevant implication. So, for example, according to this approach, the complete and immediate ground of the formula $A \rightarrow \neg \neg B$ is the multiset $\{A \rightarrow B\}$, whilst the complete and immediate ground of the formula $A \rightarrow B \wedge C$ is the multiset $\{A \rightarrow B, A \rightarrow C\}$. As Poggiolesi informally shows, all grounding principles for relevant implication that emerge from her approach satisfy pattern*.

The aim of the present work is to construct a logic of grounding containing grounding rules for relevant implication that formalize pattern* and with which one can derive Poggiolesi's grounding principles for relevant implication. In order to reach our goal, the strategy will be to draw inspiration from the logic developed in Poggiolesi (2018): indeed this logic contains a grounding rule for negation that formalizes a pattern similar to pattern*. ${ }^{5}$ Moreover, the logic that we develop in this paper has the following features. (i) Our logic will focus on the notion of complete and immediate formal grounding, leaving the study of other types of formal grounding - complete and immediate, partial and immediate and partial and mediate - for future research. (ii) It will have the form of a calculus of natural deduction and (iii) it will formalize the relation of grounding both as a meta-linguistic relation as well as an operator. (iv) Our logic will contain grounding rules for the connectives of conjunction and disjunction that are the same as those that can be found in Poggiolesi (2018); the rule for negation formulated in Poggiolesi (2018) will be extended so to generate complete and immediate grounds for the negation of relevant implications; finally new grounding rules for relevant implication will be introduced.

Note that exactly as the logic presented in Poggiolesi (2018) is proved to be the formal counterpart of the definition of complete and immediate formal grounding introduced in Poggiolesi (2016b), in the same way the logic developed in this paper will be shown to be the formal counterpart of the definition of the notion of complete and immediate formal grounding in the background of a relevant framework introduced in Poggiolesi (2020a). Finally, we will show that our logic of grounding for relevant implication enjoys a sort of deduction theorem at the grounding level: we will prove an equivalence between grounding formalized as a metalinguistic relation and grounding formalized as a connective.

The paper is organized as follows. In Section 2 we will recall the definition of complete and immediate formal grounding in the relevant framework, which our logic of grounding aims to capture; in Section 3 we will introduce the calculus $\mathcal{R G D}$, while in Section 4 we will prove soundness and completeness between the calculus $\mathcal{R G D}$ and the definition of grounding introduced in Section 2. In Section 5 we will prove that the calculus $\mathcal{R G D}$ conservatively extends the relevant logic $\mathbf{R}$, that it is consistent and that it enjoys the deduction theorem at the grounding level. Finally, in Section 6 we will draw some conclusions.

\footnotetext{
${ }^{5}$ This rule for negation says that $A$ is a (complete and immediate) ground of $\neg B$ if from $A$ and $B$ a contradiction follows (in a certain way).
} 


\section{The definition of complete and immediate ground- ing in a relevant framework}

We use this section to introduce the definition of complete and immediate formal grounding adequate for the relevant framework which has been proposed by Poggiolesi (2020a) and is the basis of the logic that we aim at developing. Let us start by introducing some necessary notions concerning the relevant system $\mathbf{R}$ (see also Anderson and Belnap (1975); Mares (2014); Dunn and Restall (2002)).

Definition 2.1. The relevant language $\mathcal{L}^{r}$ is composed of a denumerable stock of propositional atoms $(p, q, r, \ldots)$, the logical operators $\neg, \wedge, \vee$ and $\rightarrow$, the parentheses $($,$) and the square brackets [, ]. Propositional formulas are$ standardly constructed and denoted by capital letters $A, B, C, \ldots$. The set of propositional formulas so defined is denoted by $\mathbb{P F}$.

Once the relevant language $\mathcal{L}^{r}$, together with a set of propositional formulas $\mathbb{P F}$, is given, we can introduce the Hilbert system $\mathbf{R}$, whose axioms and rules are shown in Figure 2. We will write $M \vdash_{R} A$ to denote that "there is a proof in $\mathbf{R}$ that $M$ entails $A$ " in the sense of Anderson and Belnap (1975, p.277). ${ }^{6}$ An important property of the relevant system $\mathbf{R}$ linked to this notion of entailment is the Entailment theorem, namely :

Theorem 2.2. For any formula $A$ and multiset $M \in \mathcal{L}^{r}$, we have that: $M \vdash_{R} A$ if, and only if, $\vdash_{R} \wedge M \rightarrow A$

Proof. From left to right using Anderson and Belnap's theorem and the reasoning in its proof, in particular the relation with the natural deduction calculus for $\mathbf{R}$, see (Anderson and Belnap, 1975, §23.6, §27.2). From right to left, an immediate application of the rules of the Hilbert calculus.

As explained by Dunn and Restall (2002), the system $\mathbf{R}$ has both an algebraic semantics and a frame-semantics. The former has been introduced by Dunn (1970), whilst the latter has been developed by Urquhart (1972); Fine (1974) and Routley and Meyer (1973). Routley and Meyer's approach is probably the most well-known (see Mares (2014)) and it is based on the idea of interpreting the implication connective by means of a ternary relation $R i j z$ such that $i \models A \rightarrow B$ if, and only if, $\forall j, z \in W$ (if $\operatorname{Rij} z$ and $j \models A$, then $z \mid=B$ ). In this approach, while the connectives of conjunction and disjunction are treated classically, the negation connective is treated by an unary operation + on worlds ${ }^{7}$ such that for each world $i$, there is a world $i^{+}, i \models \neg A$ if, and only if, $i^{+} \not \models A .{ }^{8}$ By denoting with $\mathcal{R}^{+}$the class of relevant frames of Routley

\footnotetext{
${ }^{6}$ This notion corresponds to a derivation from $M$ to $A$ with control indexes (namely $M$ and $A$ have the same set of indexes) in the natural deduction calculus for $\mathbf{R}$, see Dunn and Restall (2002).

${ }^{7}$ We do not use the symbol $*$ that is more common in the literature because we employ it later for the notion of robust condition.

${ }^{8}$ Differently from worlds of Kripke semantics, worlds of the relevant approach can be either inconsistent or incomplete. This is due to fact that formulas as $p \wedge \neg p \rightarrow q$ or $p \rightarrow q \vee \neg q$
} 
Figure 1: Hilbert-style axiomatisation of relevant $\operatorname{logic} \mathbf{R}$

\begin{tabular}{|c|c|c|c|}
\hline $\mathrm{A} 1.1$ & $A \rightarrow A$ & $\mathrm{~A} 1.2$ & $(A \rightarrow((A \rightarrow B)) \rightarrow(A \rightarrow B)$ \\
\hline A 1.3 & $A \rightarrow((A \rightarrow B) \rightarrow B)$ & $\begin{array}{l}\mathrm{A} 1.4 \\
C))\end{array}$ & $(A \rightarrow B) \rightarrow((B \rightarrow C) \rightarrow(A \rightarrow$ \\
\hline A 2.1 & $A \wedge B \rightarrow A$ & $\mathrm{~A} 2.2$ & $A \wedge B \rightarrow B$ \\
\hline $\mathrm{A} 2.3$ & $((A \rightarrow B) \wedge(A \rightarrow C)) \rightarrow(A \rightarrow B \wedge C)$ & & \\
\hline A3.1 & $A \rightarrow A \vee B$ & A 3.2 & $B \rightarrow A \vee B$ \\
\hline A 3.3 & $(A \vee B \rightarrow C) \leftrightarrow(A \rightarrow C) \wedge(B \rightarrow C)$ & & \\
\hline A 4.1 & $\neg \neg A \rightarrow A$ & $\mathrm{~A} 4.2$ & $(A \rightarrow \neg B) \rightarrow(B \rightarrow \neg A)$ \\
\hline A5 & $((A \wedge B) \vee C) \rightarrow((A \wedge B) \vee(A \wedge C))$ & & \\
\hline MP & $A \rightarrow B, A \vdash_{R} B$ & & \\
\hline IC & $A, B \vdash_{R} A \wedge B$ & & \\
\hline
\end{tabular}

and Meyer (1973), the soundness and completeness theorem is provable for the $\operatorname{logic} \mathbf{R}$.

Theorem 2.3. For any formula $A \in \mathcal{L}^{r}$, we have that: $A$ is provable in the relevant system $\mathbf{R}$ if, and only if, $A$ is valid in the class of frames $\mathcal{R}^{+}$.

Proof. See (Dunn and Restall, 2002, p.70-77).

In what follows, in order to contain the complexity of the issue, we will restrict our attention to the complete and immediate formal grounds of those implicative formulas which contain conjunction, disjunction and negation, but are not in their turn composed of other implications. We leave the grounding analysis of these formulas for future research.

Definition 2.4. Given the set $\mathbb{P F}$ of all formulas of the language $\mathcal{L}^{r}$ we isolate the subset $\mathbb{P F} \rightarrow$ that only contains those formulas that do not contain nested implications, i.e. if there is an implication, it does not itself contain another.

The definition of the notion of grounding proposed by Poggiolesi aims at capturing grounding relations amongst the formulas $\mathbb{P F} \rightarrow$ of the logic $\mathbf{R}$ and relies on two different ideas. The first consists in organizing all formulas belonging to the set $\mathbb{P F} \rightarrow$ in a grounding hierarchy by means of the notion of $g$-complexity.

are not wanted to be proved to be valid. And for that matter, we need worlds to be able to satisfy both $p$ and $\neg p$, and to not satisfy neither $q$ nor $\neg q$. 
Of particular interest are those formulas that belong to different levels of the hierarchy and are linked by the relation of being completely and immediately less g-complex. Once all formulas are organized into the hierarchy, the task is to identify those formulas, enjoying the relation of being completely and immediately less g-complex, that also enjoy an authentic dependence between them. This authentic dependence, which is the countersign of a grounding relation, is formally captured by what Poggiolesi calls positive and negative derivability. Given a multiset of formulas $M$ and a formula $A, M$ and $A$ enjoy an authentic dependence between them when they satisfy positive and negative derivability, namely when not only $A$ is derivable from (the conjunction of each element of) $M$, but also $\neg A$ is derivable from (the conjunction of) the negation of each element of $M$.

In what follows, we will introduce all the technical ingredients needed to formulate the definition of complete and immediate formal grounding in the framework of $\mathbf{R}$ elaborated by Poggiolesi. A conceptual explanation of these technical elements can be found in Poggiolesi (2020a).

Definition 2.5. Let $D$ be a formula of $\mathbb{P F}_{\rightarrow}$. The converse of $D$, written $D^{*}$, is defined in the following way

$$
D^{*}= \begin{cases}\neg^{n-1} E, & \text { if } D=\neg^{n} E \text { and } \mathrm{n} \text { is odd } \\ \neg^{n+1} E, & \text { if } D=\neg^{n} E \text { and } \mathrm{n} \text { is even }\end{cases}
$$

where the principal connective of $E$ is not a negation, $n \geqslant 0$ and 0 is taken to be an even number. ${ }^{9}$

Here are some examples that help to clarify Definition 2.5. If $D=\neg \neg \neg \neg p$, then its converse, $D^{*}$, is $\neg \neg \neg \neg \neg p$. If $D=\neg(A \wedge B)$, then its converse, $D^{*}$, is $(A \wedge B)$; finally, if $D=(A \rightarrow B)$, then its converse, $D^{*}$, is $\neg(A \rightarrow B)$. From now on we will use capital letters to refer to formulas of the language $\mathcal{L}^{r}$ and their converse.

Definition 2.6. Consider a formula $A \in \mathbb{P F}_{\rightarrow}$. We will say that $A$ is $a$ - $c$ equiv (for associatively and commutatively equivalent) to $B$, if, and only if, $A$ can be obtained from $B$ by applications of associativity and commutativity of conjunction and disjunction.

Here are some examples of formulas which stand in the relation $a-c$ equiv. If $A$ is of the form $E \wedge F$, then the formula $F \wedge E$ is a-c equiv to it. To take another example, if $A$ is of the form $\neg((B \vee C) \wedge(D \vee F))$ the formulas $\neg((C \vee B) \wedge(D \vee F)), \neg((B \vee C) \wedge(F \vee D)), \neg((C \vee B) \wedge(F \vee D))$ are $a-c$ equiv to it. If $A$ is of the form $G \rightarrow((B \vee C) \vee(D \vee F))$, then the formulas $G \rightarrow((B \vee D) \vee(C \vee F)), G \rightarrow((D \vee B) \vee(F \vee C)), G \rightarrow((B \vee F) \vee(D \vee C))$ are all $a-c$ equiv to it.

Definition 2.7. For any $A, B \in \mathbb{P F}_{\rightarrow}, A \cong B$ if, and only if:

\footnotetext{
${ }^{9}$ Note that $\neg{ }^{0} E$ is just $E$. Also we keep the term converse for continuity with Poggiolesi's work. However, one should not confuse $*$ with an idempotent operator.
} 
$A$ is $a-c$ equiv to $B$ or $A$ is $a-c$ equiv to $B^{*}$

Definition 2.8. Given a multiset of formulas $M$ and a formula $C \in \mathbb{P F}_{\rightarrow}$, we say that $M$ is completely and immediately less $g$-complex than $C$ (in a relevant framework), if, and only if: ${ }^{10}$

- $C \cong \neg \neg B$ and $M=\{B\}$ or $M=\left\{B^{*}\right\}$, or

- $C \cong B \circ D$ and $M=\{B, D\}$, or $M=\left\{B^{*}, D\right\}$, or $M=\left\{B, D^{*}\right\}$, or $M=\left\{B^{*}, D^{*}\right\}$, or

- $C \cong \neg \neg B \rightarrow D$ or $C \cong B \rightarrow \neg \neg D$, and $M=\{B \rightarrow D\}$ or $M=$ $\{\neg(B \rightarrow D)\}$, or

- $C \cong B \circ D \rightarrow E$ and $M=\{B \rightarrow E, D \rightarrow E\}$, or $M=\{\neg(B \rightarrow E), D \rightarrow$ $E\}$, or $M=\{B \rightarrow E, \neg(D \rightarrow E)\}$, or $M=\{\neg(B \rightarrow E), \neg(D \rightarrow E)\}$, or

- $C \cong \neg(B \circ D) \rightarrow E$ and $M=\left\{B^{*} \rightarrow E, D^{*} \rightarrow E\right\}$, or $M=\left\{\neg\left(B^{*} \rightarrow\right.\right.$ $\left.E), D^{*} \rightarrow E\right\}$, or $M=\left\{B^{*} \rightarrow E, \neg\left(D^{*} \rightarrow E\right)\right\}$, or $M=\left\{\neg\left(B^{*} \rightarrow\right.\right.$ $\left.E), \neg\left(D^{*} \rightarrow E\right)\right\}$, or

- $C \cong B \rightarrow D \circ E$ and $M=\{B \rightarrow D, B \rightarrow E\}$, or $M=\{\neg(B \rightarrow D), B \rightarrow$ $E\}$, or $M=\{B \rightarrow D, \neg(B \rightarrow E)\}$, or $M=\{\neg(B \rightarrow D), \neg(B \rightarrow E)\}$, or

- $C \cong B \rightarrow \neg(D \circ E)$ and $M=\left\{B \rightarrow D^{*}, B \rightarrow E^{*}\right\}$, or $M=\{\neg(B \rightarrow$ $\left.\left.D^{*}\right), B \rightarrow E^{*}\right\}$, or $M=\left\{B \rightarrow D^{*}, \neg\left(B \rightarrow E^{*}\right)\right\}$, or $M=\{\neg(B \rightarrow$ $\left.\left.D^{*}\right), \neg\left(B \rightarrow E^{*}\right)\right\}$.

In Poggiolesi (2020a) the standard notion of logical complexity (that counts the number of connectives occurring in a formula) is substituted by the notion of $g$ complexity which represents the grounding counterpart (and more fine-grained) way of counting the complexity of a relevant formula. Although the notion of g-complexity provides deep insights into Poggiolesi's account, we do not present it in this paper since it is rather long and laborious. The important feature to underline is that the relation of being completely and immediately less g-complex introduced in the above definition is shaped by the notion of g-complexity. In particular, a multiset $M$ is completely and immediately less g-complex than a formula $C$ when it contains all those 'subformulas' of $C^{11}$ which are such that the sum of their g-complexity is immediately lower than that of $C$. Here are some examples:

- the multisets $\{(p \wedge q) \wedge r\},\{\neg((q \wedge p) \wedge r)\}$ are both completely and immediately less g-complex than the formulas $\neg \neg(r \wedge(q \wedge p))$ and $\neg \neg \neg(r \wedge$ $(q \wedge p))$

\footnotetext{
${ }^{10}$ In what follows we use the symbol $\circ$ to either denote conjunction or disjunction.

${ }^{11}$ For the notion of subformula adapted to the grounding framework see Poggiolesi (2016b, 2020a).
} 
Figure 2: Grounds for relevant implications

\begin{tabular}{|ll|}
\hline Conclusion & Complete and Immediate Formal Grounds \\
\hline$\neg \neg A \rightarrow B$ & $A \rightarrow B$ \\
$A \rightarrow \neg \neg B$ & $A \rightarrow B$ \\
$A \rightarrow B \wedge C$ & $\{A \rightarrow B, A \rightarrow C\}$ \\
$A \rightarrow \neg(B \vee C)$ & $\left\{A \rightarrow B^{*}, A \rightarrow C^{*}\right\}$ \\
$A \vee B \rightarrow C$ & $\{A \rightarrow C, B \rightarrow C\}$ \\
$\neg(A \wedge B) \rightarrow C$ & $\left\{A^{*} \rightarrow C, B^{*} \rightarrow C\right\}$ \\
& \\
where the relation of complete and immediate formal grounding is closed under ac-equivalence
\end{tabular}

- the multisets $\{(p \vee q), r\},\{\neg(p \vee q), r\},\{(p \vee q), \neg r\},\{\neg(p \vee q), \neg r\}$ are all completely and immediately less g-complex than the formulas $(p \vee q) \wedge r$ and $(q \vee p) \wedge r$, as well as than the formulas $\neg((p \vee q) \wedge r), \neg((q \vee p) \wedge r)$.

- the multisets $\{s \rightarrow p, s \rightarrow r\},\{\neg(s \rightarrow p), s \rightarrow r\},\{s \rightarrow p, \neg(s \rightarrow r)\}$, $\{\neg(s \rightarrow p), \neg(s \rightarrow r)\}$ are all completely and immediately less g-complex than the formulas $s \rightarrow p \circ r, \neg(s \rightarrow p \circ r)$, as well as the formulas $s \rightarrow r \circ p$, $\neg(s \rightarrow r \circ p)$, where $\circ=\{\wedge, \vee\}$.

Definition 2.9. For any formula $A \in \mathbb{P F}_{\rightarrow}$, and for any consistent multiset of formulas $C \cup M$ such that $C$ and $M \in \mathbb{P F}_{\rightarrow}$, we say that, under the robust condition $C$ (that may be empty), $M$ completely and immediately formally grounds $A$ in a relevant framework, in symbols $[C] M \sim{ }_{R}$ A, if and only if:

$-\bigwedge M \vdash_{R} A$,

$-C, \wedge \neg(M) \vdash_{R} \neg A$,

- $\{C, M\}$ is completely and immediately less g-complex than $A$ according to Definition 2.8.

where the notation $\bigwedge(M)$ stands for the conjunction of each element of $M$, whilst $\bigwedge \neg(M)$ stands for the conjunction of the negation of each element of $M$.

As proved in Poggiolesi (2020a), this definition of complete and immediate formal grounding conservatively extends that elaborated for the classical framework: the principles concerning negation, conjunction and disjunction of the classical framework remain the same in the relevant framework, whilst new grounding principles governing relevant implication and negation of relevant implication emerge naturally (see Figure 2 and 3, respectively ${ }^{12}$ ). Definition 2.9 involves a robust condition, namely the formula $C$ in square brackets. The notion of robust condition can be described briefly on the example of a disjunction like $A \vee B$, in a situation where the formula $A$ is true. In this case, $A$ is certainly a ground for $A \vee B$; but in order for $A$ to be the complete ground for $A \vee B$,

\footnotetext{
${ }^{12}$ Note that in the tables of Figures 2 and 3 certain expected implications, such as $A \wedge B \rightarrow C$, $\neg(A \rightarrow B \vee C), A \rightarrow \neg(B \wedge C)$, do not appear since, as explained in Poggiolesi (2020a), these implications do not have formal grounds according to Definition 2.9.
} 
Figure 3: Grounds for negation of relevant implication

\begin{tabular}{|ll|}
\hline Conclusion & Complete and Immediate \\
& Formal Grounds \\
$\neg(\neg \neg A \rightarrow B)$ & $\neg(A \rightarrow B)$ \\
$\neg(A \rightarrow \neg \neg B)$ & $\neg(A \rightarrow B)$ \\
$\neg(A \rightarrow B \wedge C)$ & $\{\neg(A \rightarrow B), \neg(A \rightarrow C)\}$ \\
& $\{\neg(A \rightarrow B)\}[A \rightarrow C]$ \\
& $\{\neg(A \rightarrow C)\}[A \rightarrow B]$ \\
& $\left\{\neg\left(A^{*} \rightarrow C\right), \neg\left(B^{*} \rightarrow C\right)\right\}$ \\
& $\left\{\neg\left(A^{*} \rightarrow C\right)\right\}\left[B^{*} \rightarrow C\right]$ \\
& $\left\{\neg\left(B^{*} \rightarrow C\right)\right\}\left[A^{*} \rightarrow C\right]$ \\
$\neg(\neg(A \wedge B) \rightarrow C) \quad\{\neg(A \rightarrow C), \neg(B \rightarrow C)\}$ \\
& $\{\neg(A \rightarrow C)\}[B \rightarrow C]$ \\
& $\{\neg(B \rightarrow C)\}[A \rightarrow C]$ \\
$\neg(A \vee B \rightarrow C)$ & $\left\{\neg\left(A \rightarrow B^{*}\right), \neg\left(A \rightarrow C^{*}\right)\right\}$ \\
& $\left\{\neg\left(A \rightarrow B^{*}\right)\right\}\left[A \rightarrow C C^{*}\right]$ \\
& $\left\{\neg\left(A \rightarrow C C^{*}\right)\right\}\left[A \rightarrow B^{*}\right]$ \\
$\neg(A \rightarrow \neg(B \vee C)) \quad$ & \\
& \\
&
\end{tabular}

it is necessary to specify that $B$ is false (i.e. that $B$ is not also a ground for $A \vee B$ ); in other terms, it is the falsity of $B$ that ensures that, or is a (robust) condition for $A$ to be the complete ground for $A \vee B$. Thus, $A$ is the complete and immediate formal ground for $A \vee B$ under the robust condition that $B$ is false. The reader is referred to Poggiolesi (2016b, 2020a,b) for a more detailed clarification of the notion of robust condition.

\section{The calculus $\mathcal{R G D}$}

Definition 3.1. The language $\mathcal{L}^{g}$ extends the relevant language $\mathcal{L}^{r}$ (see Definition 2.1) by means of the completely and immediately because connective $\triangleright$. As before, $A, B, \ldots$ denote formulas, whilst $M, N, \ldots$ denote multisets of formulas.

Definition 3.2. Let $\mathbb{B}$ be the set of completely and immediately because formulas defined in the following way:

$$
\begin{aligned}
\mathbb{B}:= & \left\{A \triangleright B \mid A, B \in \mathbb{P F}_{\rightarrow}\right\} \cup\left\{A, C \triangleright B \mid A, B, C \in \mathbb{P F}_{\rightarrow}\right\} \cup \\
& \left\{[C] A \triangleright B \mid A, B, C \in \mathbb{P F}_{\rightarrow}\right\}
\end{aligned}
$$

Note that the connective $\triangleright$ is not supposed to match the natural-language use of the term because; it is rather supposed to capture those uses of 'because' that correspond to the concept of complete and immediate formal grounding. Moreover, while in the natural language use of the term 'because' the explanans 
Figure 4: Rules of the $\mathcal{R D}$ part

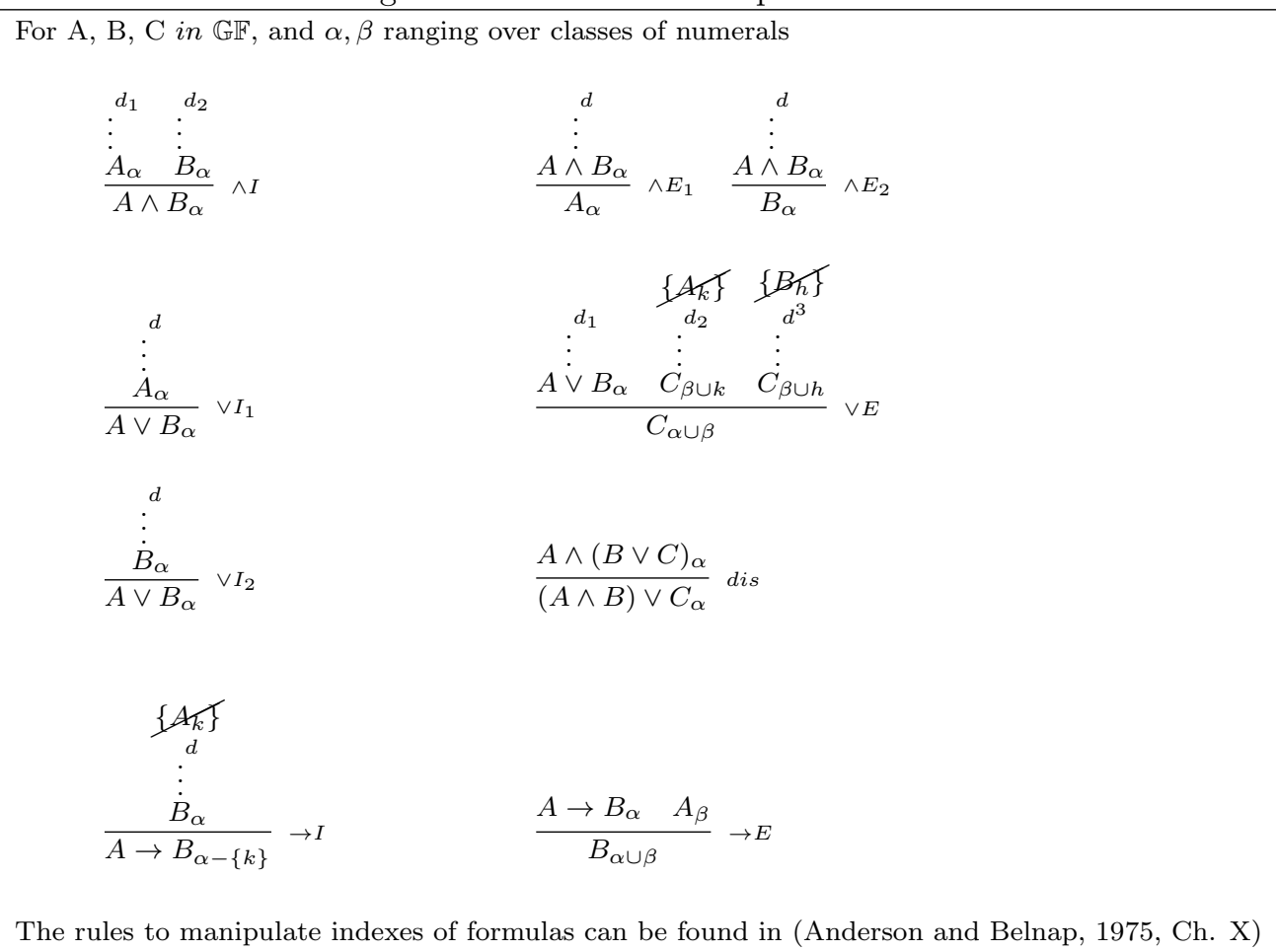

come after the 'because' and the explanandum before, in the connective $\triangleright$ these positions are inverted. We will denote with $\mathbb{G F}$ the closure of $\mathbb{P F} \cup \mathbb{B}$ under the connectives of negation, conjunction, disjunction of $\mathcal{L}^{g}$.

Based on the language $\mathcal{L}^{g}$, we will construct the calculus $\mathcal{R G D}$, which will be our logic of grounding in the relevant framework $\mathbf{R}$. The calculus $\mathcal{R G D}$ can be thought of as divided in three parts:

(i) the first part, the $\mathcal{R D}$ part, which is the basis of the calculus, is just the natural deduction calculus for relevant logic formulated in the language $\mathcal{L}^{g}$. It is composed of introduction and elimination rules with indexes that characterize the relevant relation of derivability, denoted by $\vdash_{\mathcal{R D}}$.

(ii) the second part of the calculus, the $\mathcal{G}$ part formulated for the set of formulas $\mathbb{P F}_{\rightarrow}$, is where the grounding concept is formalized as a meta-linguistic relation. This meta-linguistic relation is denoted by the symbol $\mid \sim^{13}$ and is called (complete and immediate) formal explanation. One can think of

\footnotetext{
${ }^{13}$ Note that we use the same symbol for the relation of complete and immediate formal explanation and the relation of complete and immediate formal grounding, introduced in Definition 2.9. Though this might at first appear confusing, both notions are syntactic and will be proved to be equivalent, so we prefer to leave the notation as it is. This is analogous
} 
it as the grounding analogue of the meta-linguistic relation of derivability. Hence, as derivability is definable via (inferential) rules, in the same way the relation of formal explanation will be defined via grounding rules which will be introduced shortly.

(iii) the third part of the calculus, the $\mathcal{D}$ part, is based on the language $\mathcal{L}^{g}$ and it is where the connective $\triangleright$ is proof-theoretically defined by means of one introduction rule and three elimination rules. The introduction rule naturally provides a link between parts (i) and (ii) of the calculus. Thanks to the $\mathcal{D}$ part of the calculus, the relation $\mid \simeq$, that holds for the whole logic $\mathcal{R G D}$ and which combines the relevant notion of derivability with the notion of complete and immediate formal explanation, can finally be defined.

For the sake of clarity, we will introduce each part separately. Let us start by (i). The $\mathcal{R D}$ part of the calculus is composed by the rules of Figure 4, which are the rules of the natural deduction calculus for the relevant logic $\mathbf{R}$ (see Dunn and Restall (2002)). As it is standard, in these rules the curly brackets stand for the discharge of several formulas at the same time. The set of derivations $\mathbb{R D}$ that can be constructed by means of the rules of $\mathcal{R D}$ is the standard one, see Dunn and Restall (2002). We write $M_{\alpha} \vdash_{\mathcal{R D}} A_{\alpha}$ to denote the fact that there exists a natural deduction derivation from the multiset $M$ to $A$ in $\mathcal{R D}$, where the multiset $M$ and the formula $A$ have the same set of indexes. The Hilbert system $\mathbf{R}$ and the natural deduction calculus $\mathcal{R D}$ prove the same formulas, as the following theorem shows.

Theorem 3.3. For any formula $A$ and multiset $M \in \mathbb{G F}$ and mutliset of labels $\alpha$, we have that: $M \vdash_{R} A$ if, and only if, $M_{\alpha} \vdash_{\mathcal{R D}} A_{\alpha}$, where $M_{\alpha}$ stands for the multiset of formulas $B_{k}^{n}$, such that each $B$ belongs to $M$ and each $k$ belongs to $\alpha$.

Proof. See (Dunn and Restall, 2002, p. 20).

In order to introduce part (ii) of the calculus, we need some preliminary notions and definitions.

Definition 3.4. Given $A \in \mathbb{P F}_{\rightarrow}$, let $\mathbb{A} \mathbb{C}(A)$ be the set of all elements $B$ such that $A$ is a-c equiv to $B$ (Definition 2.6). From now on we will use $\mathbf{A}, \mathbf{B}, \mathbf{C}$, ... to denote generic elements belonging to the set $\mathbb{A} \mathbb{C}(A), \mathbb{A} \mathbb{C}(B), \mathbb{A} C(C), \ldots$ respectively.

Definition 3.5. Let $\mathbf{A}$ be a generic element belonging to the set $\mathbb{A} \mathbb{C}(A)$. Then $\mathbf{A}^{*}$ denotes generic elements of $\mathbb{A} \mathbb{C}\left(A^{*}\right)$.

Let us illustrate our notation with some examples. Consider an object $A$ of the form $s \vee t \rightarrow \neg((p \wedge q) \vee r)$, then

to what happens in Hilbert systems and Gentzen systems: they share the same symbol for derivability. 
A denotes any of the following objects: $s \vee t \rightarrow \neg((p \wedge q) \vee r), t \vee s \rightarrow \neg((q \wedge p) \vee r)$, $t \vee s \rightarrow \neg(r \vee(p \wedge q)), \neg(r \vee(q \wedge p))$

$\mathbf{A}^{*}$ denotes any of the following objects: $\neg(s \vee t \rightarrow(p \wedge q) \vee r), \neg(s \vee t \rightarrow$ $(q \wedge p) \vee r), \neg(s \vee t \rightarrow r \vee(p \wedge q), r \vee(q \wedge p))$

From now on, capital letters of bold character will be used to refer to both $\mathbf{A}, \mathbf{B}, \mathbf{C}, \ldots$ but also $\mathbf{A}^{*}, \mathbf{B}^{*}, \mathbf{C}^{*}, \ldots \mathbf{M}, \mathbf{N}, \ldots$ will be multisets of capital letters of bold character.

Let us now move to the grounding rules for the $\mathcal{G}$ part of the calculus $\mathcal{R G D}$. As for the grounding rules for conjunction and disjunction, these are the same as

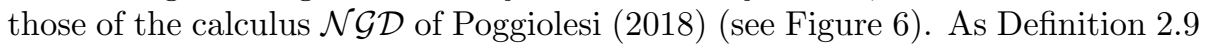
correctly captures, the complete and immediate formal grounds of conjunction and disjunction do not change passing from classical logic to relevant logic $\mathbf{R}$. Hence the grounding rule of conjunction says that the complete and immediate formal grounds of a conjunction are its conjuncts. The grounding rules for disjunction are three: the former says that the complete and immediate formal grounds of a disjunction are its disjuncts; the other two say that the complete and immediate formal grounds of a disjunction is one disjunct under the robust condition that the converse of the other disjunct is true. A simple way of expressing these rules is the following: ${ }^{14}$

$$
\begin{aligned}
& \frac{A \quad B}{\overline{A \wedge B}}
\end{aligned}
$$

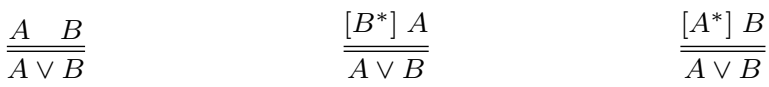

However the relation of grounding that we aim at capturing is closed under ac-equivalence. In order to take care of this important feature and yet not lose the uniqueness of our rules, we use the bold letters introduced before. So we slightly change grounding rules for conjunction and disjunction in the following way:

$$
\begin{array}{ll}
\frac{\mathbf{A} \mathbf{B}}{\overline{\mathbf{A} \wedge \mathbf{B}} \wedge G} \\
\frac{\mathbf{A} \mathbf{B}}{\overline{\mathbf{A} \vee \mathbf{B}} \vee G_{1}} \quad \frac{\overline{\left[\mathbf{B}^{*}\right] \mathbf{A}}}{\overline{\mathbf{A} \vee \mathbf{B}} \vee G_{2}} \quad \frac{\overline{\left[\mathbf{A}^{*}\right] \mathbf{B}}}{\overline{\mathbf{A} \vee \mathbf{B}} \vee G_{3}}
\end{array}
$$

The grounding rule for conjunction written in the bold notation not only allows us to say that, i.e. $p \wedge q$ and $r$ formally explain $(p \wedge q) \wedge r$, but that they also explain $(q \wedge p) \wedge r, q \wedge(p \wedge r), r \wedge(p \wedge q)$ and so on.

Let us now move to the grounding rules for negation and relevant implication. In order to introduce these grounding rules, we need inference rules since in

${ }^{14}$ Note that to aid readability, we adopt a new notation with respect to Poggiolesi (2018). 
both of them the grounds are established by means of what can be inferred from them: in the case of the grounding rule for negation a contradiction, in the case of the grounding rule for implication, the consequent of the implication itself. We therefore introduce a new notation $<\left(R ; R^{\prime}\right), R^{\prime \prime}>$ that we call combination of rules, and which denotes the fact that two rules $R$ and $R^{\prime}$ are used in a parallel way and then followed by the rule $R^{\prime \prime}$. Let us provide some examples of combinations of rules so to make clear this new notation. Consider the combination of rules $<\left(\wedge E_{1} ; \wedge E_{2}\right), \wedge I>$; it corresponds to the following inferential steps:

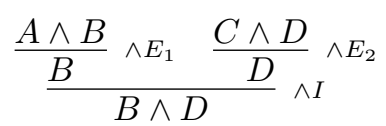

The combination of rules $<(\rightarrow E ; \rightarrow E), \vee I>$ corresponds to the following inferential steps:

$$
\frac{A \vee B \frac{A \quad A \rightarrow C}{C} \rightarrow E \frac{\not B \quad B \rightarrow C}{C} \vee I}{C} \rightarrow E
$$

Note that there might also be combinations of rules where in brackets we only have one rule, i.e. combinations of rules of the following form $<(R), R^{\prime}>$ that in a simpler way we will write $\left\langle R, R^{\prime}\right\rangle$. $\left\langle R, R^{\prime}\right\rangle$ stands for the fact that the rules $R$ and $R^{\prime}$ are used one after the other. So for example the combination of rules $<\wedge I, \vee I\rangle$ corresponds to the following inferential steps:

$$
\frac{\frac{A_{1} \quad B_{1}}{A \wedge B_{1}} \wedge I}{(A \wedge B) \vee C_{1}} \vee I
$$

whilst the following combination of rules $<\wedge E, \rightarrow E>$ corresponds to the following inferential steps:

$$
\frac{(A \wedge(B \rightarrow C))_{1}}{\frac{(B \rightarrow C)_{1}}{C_{1}} \quad B_{1}} \rightarrow E
$$

In order to simplify our task, in combination of rules we will use the rules of Figure 5 that are all straightforwardly relevantly derivable, as well as the rules of relevant deduction, both without indexes. Note that the absence of indexes is motivated by the ambition of simplifying derivations and it should not be a source of worry: in the next Section we will show that any combination of rules that we will take into account gives rise to a relevant derivation.

Definition 3.6. We will say that the multiset $M$ is completely and immediately contradictory when $M$ and $\perp$ are linked by one of these combinations of rules: 
Figure 5: Derivable rules in the calculus $\mathbb{R D}$

\begin{tabular}{|c|c|}
\hline For $\mathrm{A}, \mathrm{B}, \mathrm{C}$ in $\mathbb{G F}$ & \\
\hline$\frac{A \quad A^{*}}{\perp} \perp^{\star} I$ & \\
\hline$\frac{A}{\neg \neg A} \neg \neg I$ & $\frac{\neg \neg A}{A} \neg \neg E$ \\
\hline$B^{*} C^{*}$ & \\
\hline$\neg(B \wedge C) \quad \dot{D} \quad \dot{D}$ & $B^{*} \quad C^{*}$ \\
\hline $\bar{D} \neg \wedge \wedge$ & $\overline{\neg(B \wedge C)} \neg \vee$ \\
\hline$\frac{\neg \neg A \rightarrow B}{A \rightarrow B} \neg \neg \rightarrow$ & $\frac{A \rightarrow \neg \neg B}{A \rightarrow B} \rightarrow \neg \neg$ \\
\hline$\frac{A \rightarrow B_{1} \wedge B_{2}}{A \rightarrow B_{i}} \rightarrow \wedge_{i}$ & $\frac{A_{1} \vee A_{2} \rightarrow B}{A_{i} \rightarrow B} \vee_{i} \rightarrow$ \\
\hline$\frac{A_{i} \rightarrow \neg\left(B_{1} \vee B_{2}\right)}{A \rightarrow B_{i}^{*}} \rightarrow \neg \vee i$ & $\frac{\neg\left(A_{1} \wedge A_{2}\right) \rightarrow B}{A_{i}^{*} \rightarrow B} \neg \neg_{i} \rightarrow$ \\
\hline where $i=\{1,2\}$ & \\
\hline$-\perp I,{ }^{15}$ & \\
\hline$-<X, \perp^{*} I>$ & \\
\hline$-<Y, \perp I>$ & \\
\hline$<\left(\perp^{*} I ; \perp^{*} I\right), \vee E>$ & \\
\hline
\end{tabular}

where $X=\left\{\wedge E_{i}\right\}$ and $Y=\left\{\neg \neg \rightarrow, \rightarrow \neg \neg, \rightarrow \wedge_{i}, \vee_{i} \rightarrow, \rightarrow \neg \vee_{i}, \neg \wedge_{i} \rightarrow\right\}$. We will use the following notation to indicate that the multiset $M$ is completely and immediately contradictory:

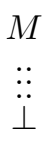

Consider two completely and immediately contradictory multisets $M$ and $N$; if the derivation from $M$ to $\perp$ contains one of these rules $\left\{\wedge E_{i}, \rightarrow \wedge_{i}, \vee_{i} \rightarrow\right.$,

\footnotetext{
${ }^{15} \mathrm{By} \perp I$ we denote those instances of the rule $\wedge I$ where the conclusion is a contradiction.
} 
$\left.\rightarrow \neg \vee_{i}, \neg \wedge_{i} \rightarrow\right\}$, whilst the corresponding derivation from $N$ to $\perp$ contains one of the following $\left\{\wedge E_{j}, \rightarrow \wedge_{j}, \vee_{j} \rightarrow, \rightarrow \neg \vee_{j}, \neg \wedge_{j} \rightarrow\right\}$, where $i=1,2$ and $j$ $=1,2$ such that $i \neq j$, then $M$ and $N$ will be said to be two complementary completely and immediately contradictory multisets. The following notation denotes this fact:

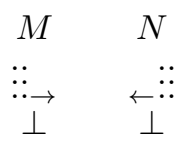

Let us provide some examples of completely and immediately contradictory multisets. The multiset $\{\neg \neg A \rightarrow B, \neg A \rightarrow B\}$ is completely and immediately contradictory since we have:

$$
\frac{\frac{\neg \neg A \rightarrow B}{A \rightarrow B} \neg \neg \rightarrow \quad \neg(A \rightarrow B)}{\perp} \perp I
$$

Also the multiset $\left\{A \vee B, A^{*}, B^{*}\right\}$ is completely and immediately contradictory since we have:

$$
\frac{A \vee B \frac{A, A^{*}}{\perp} \perp^{*} I \frac{\not B, B^{*}}{\perp} \perp^{* * I}}{\perp}
$$

Let us now provide some examples of complementary completely and immediately contradictory multisets. Consider the multiset $M:=\left\{A \wedge B, A^{*}\right\}$ as well as the multiset $N:=\left\{A \wedge B, B^{*}\right\}$. Each of them is completely and immediately contradictory since we have, respectively:

$$
\frac{\frac{A \wedge B}{A} \wedge E_{1} \quad A^{*}}{\perp} \perp{ }^{* I} \quad \frac{\frac{A \wedge B}{B} \wedge E_{2} \quad B^{*}}{\perp} \perp{ }^{*} I
$$

Moreover they are complementary since in one derivation the rule $\wedge E_{1}$ is used, whilst in the other derivation the rule $\wedge E_{2}$ is used.

Consider the multiset $M:=\left\{A \rightarrow B_{1} \wedge B_{2}, \neg\left(A \rightarrow B_{1}\right)\right\}$ as well as the multiset $N:=\left\{A \rightarrow B_{1} \wedge B_{2}, \neg\left(A \rightarrow B_{2}\right)\right\}$. Each of them is completely and immediately contradictory since we have, respectively:

$$
\frac{\frac{A \rightarrow B_{1} \wedge B_{2}}{A \rightarrow B_{1}} \rightarrow_{\wedge_{1}} \neg\left(A \rightarrow B_{1}\right)}{\perp} \perp I \quad \frac{\frac{A \rightarrow B_{2} \wedge B_{2}}{\frac{A \rightarrow B_{2}}{\perp} \rightarrow \wedge_{1} \quad \neg\left(A \rightarrow B_{2}\right)}}{\perp} \perp I
$$

Moreover they are complementary since in one derivation the rule $\rightarrow \wedge_{1}$ is used, whilst in the other derivation the rule $\rightarrow \wedge_{2}$ is used. 
Figure 6: Rules of the $\mathcal{G}$ part

For $\mathrm{A}, \mathrm{B}, \mathrm{C}$ in $\mathbb{P F}_{\rightarrow}$,

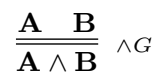

\begin{tabular}{|c|c|c|}
\hline A B & {$\left[\mathbf{B}^{*}\right] \mathbf{A}$} & {$\left[\mathbf{A}^{*}\right] \mathbf{B}$} \\
\hline$\overline{\overline{\mathbf{A} \vee \mathbf{B}}}{ }^{\vee} G_{1}$ & $\overline{\overline{\mathbf{A} \vee \mathbf{B}}} \vee G_{2}$ & $\overline{\bar{A} \vee \mathbf{B}}$ \\
\hline
\end{tabular}

$\{A, M\}$

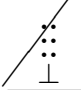

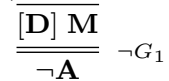

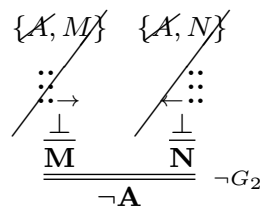

$\{A, M\},\{A, N\}$

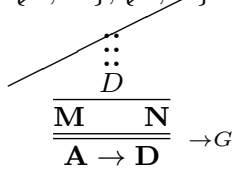

All rules carry the proviso that their premisses are consistent.

Definition 3.7. We will say that a formula $A$ is completely and immediately derivable from the multisets $M, N$, one of which might be empty, when $M, N$ and $A$ are linked by one of these combinations of rules:

- $<\rightarrow E, \neg \neg I>$,

$-<\neg \neg E, \rightarrow E>$,

- $<(\rightarrow E ; \rightarrow E), X>$

where $X=\{\wedge I, \neg \wedge E, \neg \vee, \vee E\}$. The notation:

$$
\begin{gathered}
M, N \\
: \because \\
\ddot{A}
\end{gathered}
$$

serves to indicate that $A$ is completely and immediately derivable from $M, N$.

Let us provide few examples of formulas that are completely and immediately derivable from multisets of formulas. Consider the formula $\neg \neg B$ that is completely and immediately derivable from the multiset $\{A, A \rightarrow B\}$. Indeed we have: 


$$
\frac{A \quad A \rightarrow B}{\frac{B}{\neg \neg B} \neg \neg I} \rightarrow E
$$

Consider the formula $B \wedge C$ that is completely and immediately derivable from the multisets $\{A, A \rightarrow B\}$ and $\{A, A \rightarrow C\}$. Indeed we have:

$$
\frac{A \quad A \rightarrow B}{\frac{B \quad}{B \wedge C \quad} \quad \frac{A \quad A \rightarrow C}{C} \wedge I} \rightarrow E
$$

We now have all elements to introduce part (ii) of the calculus, namely the $\mathcal{G}$ part. The $\mathcal{G}$ part is composed of the rules of Figure 6. The rule describing the complete and immediate formal explanans of a conjunction is $\wedge G$, whilst the three rules providing the complete and immediate formal explanans of a disjunction are $\vee G_{1}, \vee G_{2}, \vee G_{3}$, respectively. The rules concerning negation are $\neg G_{1}$ and $\neg G_{2}$ : in the former one, if in the inferential part there is an occurrence of one of these rules $\left\{\wedge E_{i}, \rightarrow \wedge_{i}, \vee_{i} \rightarrow, \rightarrow \neg \vee_{i}, \neg \wedge_{i} \rightarrow\right\}$, then a robust condition constituted by the formula that could have been inferred by one of the corresponding rules $\left\{\wedge E_{j}, \rightarrow \wedge_{j}, \vee_{j} \rightarrow, \rightarrow \neg \vee_{j}, \neg \wedge_{j} \rightarrow\right\}$ (where $i$ $=1,2$ and $j=1,2$ such that $i \neq j$ ), should be added in the premisses of the formal explanation. Moreover, the formula $A$ to discharge is the one that allows an increase of g-complexity. Let us give two formal explanations involving this rule:

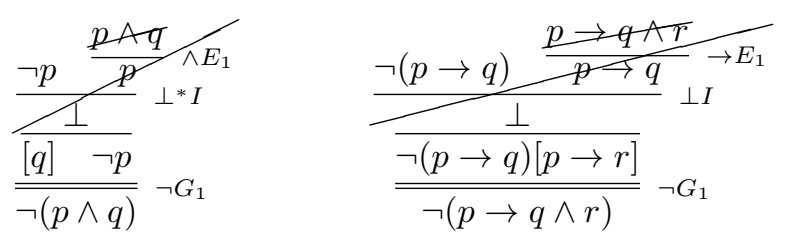

The explanatory rule for (relevant) implication is $\rightarrow G$. This rules formalizes the reasoning that we have introduced at the beginning of this paper: it indeed tells us that grounds of a formula of the form $A \rightarrow D$ are the multisets $\mathrm{M}$ and $\mathrm{N}$ (where one of the two might be empty), such that from $A$ and $M$ and $N$, the formula $D$ can be derived in a complete and immediate way.

Definition 3.8. The set of complete and immediate formal explanations of the calculus $\mathcal{R G D}$ is the smallest set $\mathbb{F E}$ of single occurrences of the rules of Figure 6 . We will write $[\mathbf{C}] \mathbf{M} \mid \sim \mathbf{A}$ for: under the robust condition $\mathbf{C}$ there exists a complete and immediate formal explanation of $\mathbf{A}$ from $\mathbf{M}$. We will also use the notation

\section{[C] $\mathbf{M}$}

to indicate that $\mathbf{A}$ is linked to $[\mathbf{C}] \mathbf{M}$ by means of one of the rules of Figure 6 . 
Let us provide some examples of complete and immediate formal explanations. The following is a complete and immediate formal explanation of a conjunctive formula:

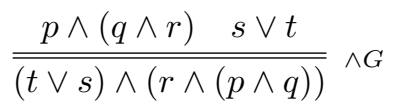

The following two are complete and immediate formal explanations of disjunctive formulas:

$$
\frac{[\neg(p \vee t)] \quad q \vee r}{\overline{(p \vee q) \vee(r \vee t)} \vee G_{2}} \quad \frac{[t \vee s] q \vee r}{\neg(t \vee s) \vee(r \vee q)} \vee G_{3}
$$

We now introduce two formal explanations of formulas in a negative form:

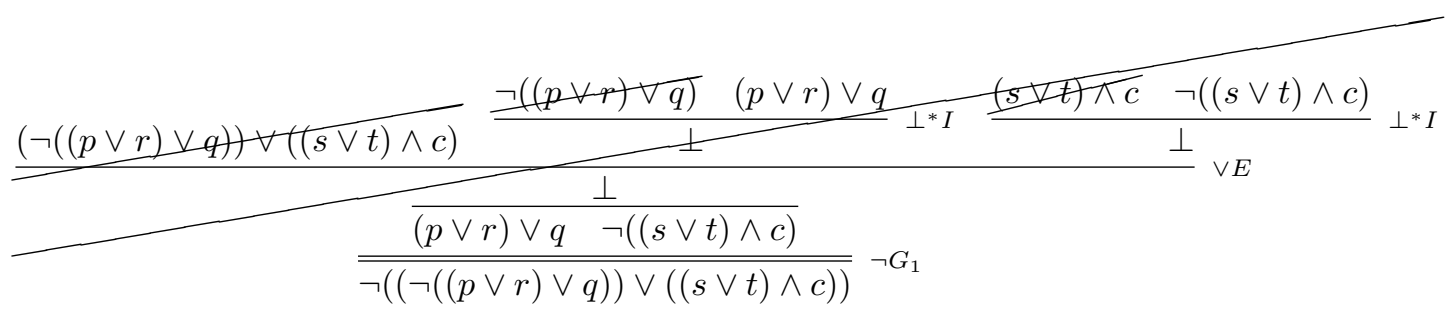

$$
\frac{\frac{\neg((p \wedge r) \wedge s \rightarrow(q \vee t)) \frac{(p \wedge r) \wedge s \rightarrow \neg \neg(q \vee t)}{(p \wedge r) \wedge s \rightarrow(q \vee t)}}{\perp} \rightarrow \neg \neg}{\frac{\perp}{\neg(r \wedge(p \wedge s) \rightarrow(q \vee t))}} \neg \neg G_{1}
$$

We finally show two formal explanations that involve (relevant) implication:

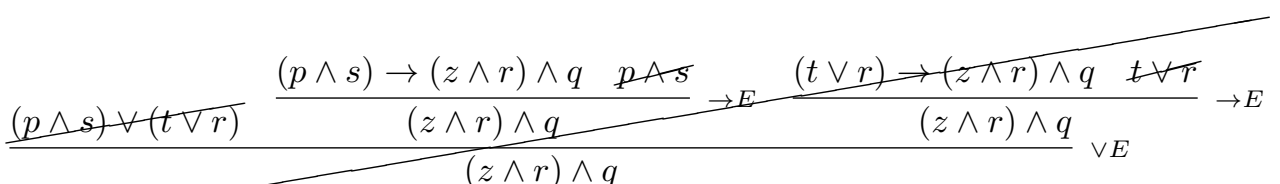

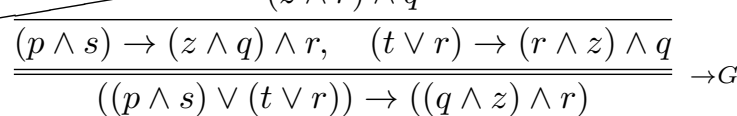

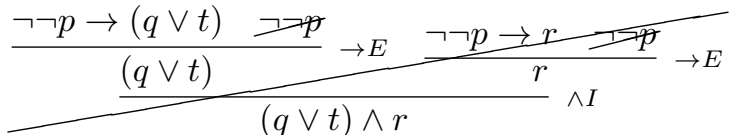

$$
\begin{aligned}
& \frac{\overline{\neg \neg p \rightarrow(q \vee t), \quad \neg \neg p \rightarrow r}}{\neg \neg p \rightarrow((q \vee t) \wedge r)} \rightarrow G
\end{aligned}
$$


Since in this paper we are only interested in a logic for the notion of complete and immediate formal ground, our grounding rules cannot form grounding chains. We leave the task of generalizing our approach to grounding chains for future research. By now, let us emphasize some important structural properties of the relation of complete and immediate formal explanation.

Lemma 3.9. The relation $\mid \sim$ is not reflexive, nor monotonic, nor transitive, nor symmetric.

Proof. The fact that the relation $\mid \sim$ is not reflexive and not symmetric are evident. The relation $\mid \sim$ is non-monotonic because there is no (admissible) weakening rule at the grounding level. Finally the relation $\mid \sim$ is not transitive because no grounding chain can be constructed by Definition 3.8.

We thus have introduced the first two parts of the calculus $\mathcal{R G D}$ : the $\mathcal{R} \mathcal{D}$ part with the related notion of relevant derivability and the $\mathcal{G}$ part with the related crucial notion of formal explanation. We now introduce the third and last part of the calculus $\mathcal{R G D}$ which is characterized by the connective $\triangleright$ and represents the bridge between the first two parts.

The $\mathcal{D}$ part of the calculus $\mathcal{R} \mathcal{G} \mathcal{D}$ is composed by the rules that introduce and eliminate the connective $\triangleright$ (see Figure 7) which represents the linguistic counterpart of the relation of complete and immediate formal explanation and which should be read as completely and immediately because. Let us spend some words clarifying these rules. As concerns the rule $\triangleright I$, let us note the similarity with the rule that standardly introduces the implication connective, namely

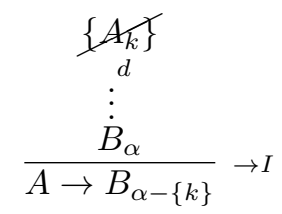

As the rule $\rightarrow I$ states that, if there exists a derivation from $A_{k}$ to $B_{\alpha}$, then $A \rightarrow B_{\alpha-\{k\}}$ can be inferred, in the same way, the rule $\triangleright I$ states that, if there exists a complete and immediate formal explanation from $M$ to $A$, then $M \triangleright A$ can be inferred (and no index needs to be attached to $M \triangleright A$ since it is a theorem). In other terms, this similarity reflects the analogy between the relation between relevant derivability and relevant implication on the one hand, and the relation between formal explanation and 'because' on the other hand.

Let us now turn to the three elimination rules that concern the connective $\triangleright$. Taken together, these rules give a precise interpretation of the connective $\triangleright$. They say that if, under the robust condition $C, A$ completely and immediately because of $M$, then: (i) the conjunction of each element of $M$ implies $A$, (ii) the conjunction of the negation of each element of $M$ together with $C$ implies the negation of $A$; (iii) $C \cup M$ is a consistent multiset and is completely and immediately less g-complex than $A$. Conditions (i)-(iii) reflect at the linguistic level the three conditions that characterize the notion of formal explanation introduced in Definition 2.9. In particular, (i) corresponds to positive derivability, (ii) to 
Figure 7: Rules of the $\mathcal{D}$ part

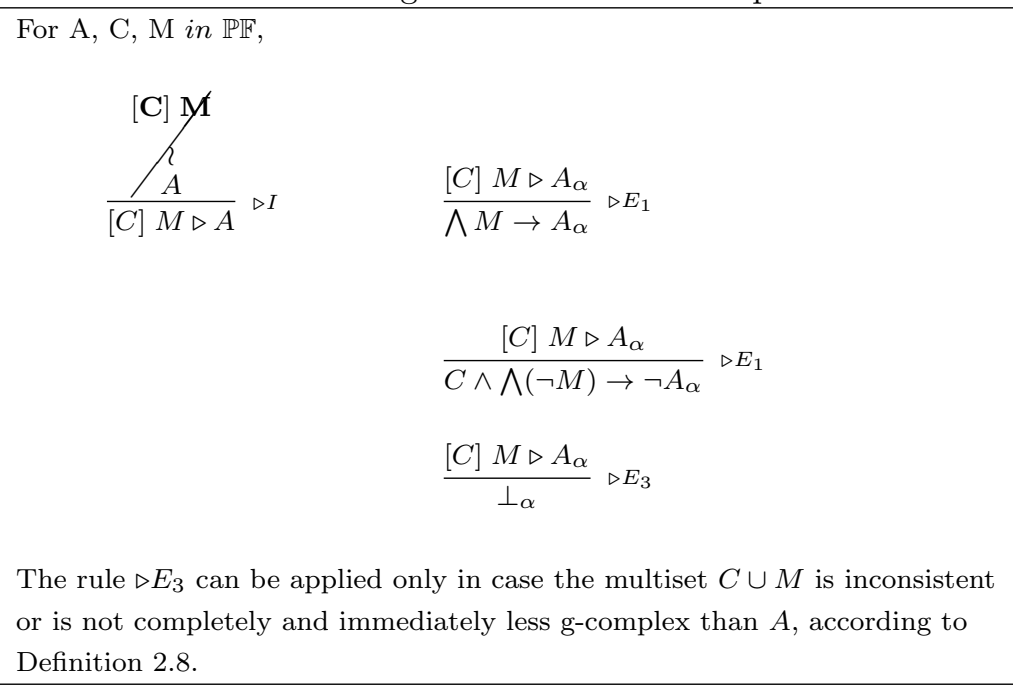

negative derivability, and (iii) is basically the same condition as complexity (and consistency).

The calculus $\mathcal{R G D}$ is thus composed of the rules of Figure 4, the rules of Figure 6 and the rules of Figure 7. The notion of derivability that holds for the whole calculus $\mathcal{R G D}$ can be denoted in the following way $M \mid \simeq A$ for: there exists a derivation belonging to $\mathcal{R G D}$ from the multiset of formulas $M$ to the formula $A$. The definition of the relation $\mid \simeq$ is easy but tedious, so we omit it here and we refer the interested reader to Poggiolesi (2018). We denote the set of derivations relative to the relation $\mid \simeq$ with $\mathbb{R} \mathbb{G} \mathbb{D}$.

\section{Soundness and Completeness}

In the last two sections we have introduced the calculus $\mathcal{R G D}$ together with its derivability relation $\mid \simeq$ and its set of derivations $\mathbb{R} G \mathbb{D}$. The calculus $\mathcal{R G D}$ is composed of three parts: first we have the $\mathcal{R} \mathcal{D}$ part of the calculus together with the relevant derivability relation $\vdash_{R}$ and the set of relevant derivations $\mathbb{R D}$; secondly, we have the $\mathcal{G}$ part of the calculus together with the formal explanation relation $\mid \sim$ and the set of formal explanations $\mathbb{F E}$; finally, we have the $\mathcal{D}$ part of the calculus that serves to define the operator $\triangleright$, connects the first two parts $\mathcal{R D}$ and $\mathcal{G}$ and allows for a combination of formal explanation and derivability in the rule $\triangleright I$.

In this section we will focus on the $\mathcal{G}$ part of the calculus and we will prove that this part is sound and complete with respect to the notion of complete and immediate formal grounding that has been introduced in Definition 2.9. In other words, in this section we will show that the grounding part of our logic 
$\mathcal{G}$ is the adequate formal counterpart of the conception of grounding that has been put forward in Poggiolesi (2020a). This result straightforwardly implies that the rule $\rightarrow G$ of the $\mathcal{G}$ part of $\mathcal{R G D}$ validate all and only those grounding principles for relevant implication that occur in Figure 2. The rules $\neg G_{1}$ and $\neg G_{2}$ of the $\mathcal{G}$ part of $\mathcal{R G \mathcal { D }}$ validates all and only those grounding principles for double negation, negation of conjunction, negation of disjunction and negation of relevant implication (see Figure 3) that emerge from Definition 2.9.

Lemma 4.1. For any composition of rules linking $\{A, M\}$ and $\perp$ in such a way that $\{A, M\}$ is completely and immediately contradictory (see Definition 3.6), it holds that, not only $A, \bigwedge M \vdash_{R} \perp$, but also $D, \neg A, \bigwedge \neg(M) \vdash_{R} \perp$, where $D$ is the formula that could have been inferred by one of these rules $\left\{\wedge E_{j}, \rightarrow \wedge_{j}\right.$, $\left.\vee_{j} \rightarrow, \rightarrow \neg \vee_{j}, \neg \wedge_{j} \rightarrow\right\}$, if the corresponding one amongst the following rules $\left\{\wedge E_{i}, \rightarrow \wedge_{i}, \vee_{i} \rightarrow, \rightarrow \neg \vee_{i}, \neg \wedge_{i} \rightarrow\right\}$ (where $i=1,2$ and $j=1,2$ such that $i \neq j$ ) has actually been used to derive the contradiction. Moreover the multiset $\{D, M\}$ is completely and immediately less g-complex than $\neg A$ according to the notion of g-complexity introduced in Definition 2.8.

Proof. We prove this lemma by distinguishing the several forms that the derivation from $\{A, M\}$ to $\perp$ might have (see Definition 3.6) and thus the several types of multiset that $\{A, M\}$ might be. For the cases where the compositions of rules are the following $\perp I,\left\langle\left(\perp^{*} I ; \perp^{*} I\right), \vee E\right\rangle,\left\langle\wedge E_{i}, \perp^{*} I\right\rangle$, the proof is analogous to the one developed in Poggiolesi (2018). We thus analyze the remaining cases.

(1) $\mathrm{d}:=\langle\neg \neg \rightarrow, \perp I>$ or $\mathrm{d}:=\langle\rightarrow \neg \neg, \perp I>$, and it has one of these two forms,

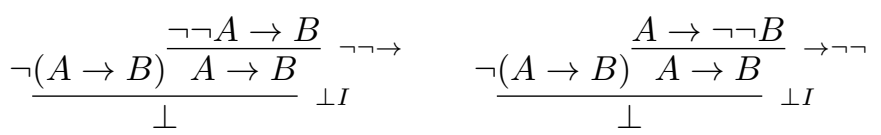

Hence our multiset is either $\{\neg(A \rightarrow B), \neg \neg A \rightarrow B\}$ or $\{\neg(A \rightarrow B), A \rightarrow \neg \neg B\}$. In both cases, a contradiction can be relevantly derived in the system $\mathbf{R}$ (also via Theorem 3.3). But it is evident that the following derivations hold as well: $\neg \neg(A \rightarrow B), \neg(\neg \neg A \rightarrow B) \vdash_{R} \perp$ and $\neg \neg(A \rightarrow B), \neg(A \rightarrow \neg \neg B) \vdash_{R} \perp$. Finally $\{\neg(A \rightarrow B)\}$ is completely and immediately less g-complex than $\neg(\neg \neg A \rightarrow B)$ but also than $\neg(A \rightarrow \neg \neg B)$ according to Definition 2.8.

(2) $\mathrm{d}:=<\rightarrow \wedge_{i}, \perp I>$ or $\mathrm{d}:=<\rightarrow \neg \vee_{i}, \perp I>$ and it has one of these two forms,

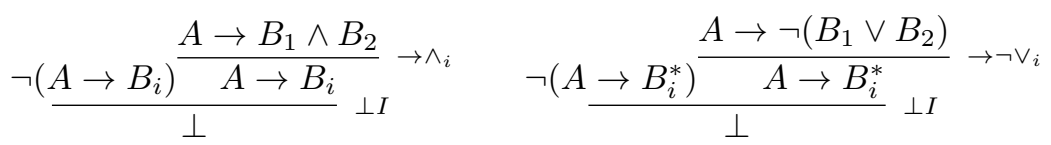


Figure 8: The calculus $\mathcal{R G D}$

$$
\mathcal{R G D}, \mid \simeq, \mathbb{R} G \mathbb{D}=\left\{\begin{array}{l}
\mathcal{R D}, \vdash, \mathbb{R D} \\
\mathcal{G}, \mid \sim, \mathbb{E} \mathbb{E} \\
\mathcal{D}
\end{array}\right.
$$

Hence our multiset is either $\left\{\neg\left(A \rightarrow B_{i}\right), A \rightarrow B_{1} \wedge B_{2}\right\}$ or $\left\{\neg\left(A \rightarrow B_{i}^{*}\right), A \rightarrow\right.$ $\left.\neg\left(B_{1} \vee B_{2}\right)\right\}$. We analyze the first multiset and derivation; the analysis of the other can be developed along analogous lines. First of all it is straightforward to see that $\neg\left(A \rightarrow B_{i}\right), A \rightarrow B_{1} \wedge B_{2} \vdash_{R} \perp$ (also via Theorem 3.3). But it is also evident that the following derivation can be constructed as well: $\neg \neg(A \rightarrow$ $\left.B_{i}\right), A \rightarrow B_{j}, \neg\left(A \rightarrow B_{1} \wedge B_{2}\right) \vdash_{R} \perp$, where $j$ is the number not denoted by $i$ amongst $\{1,2\}$. Finally $\left\{\neg\left(A \rightarrow B_{i}\right), A \rightarrow B_{j}\right\}$ is completely and immediately less g-complex than $\neg\left(A \rightarrow B_{i} \wedge B_{2}\right)$ according to Definition 2.8.

(3) $\mathrm{d}:=\left\langle\vee_{i} \rightarrow, \perp I>\right.$ or $\mathrm{d}:=\left\langle\neg \wedge_{i}, \rightarrow, \perp I>\right.$ and it has one of these two forms,

$$
\neg \frac{\left(A_{i} \rightarrow B\right) \frac{A_{1} \vee A_{2} \rightarrow B}{A_{i} \rightarrow B}}{\perp} \vee_{i} \rightarrow \quad \neg\left(A_{i}^{*} \rightarrow B\right) \frac{\neg\left(A_{1} \wedge A_{2}\right) \rightarrow B}{A_{i}^{*} \rightarrow B} \neg^{\prime} \perp \wedge_{i} \rightarrow
$$

Hence our multiset is either $\left\{\neg\left(A_{i} \rightarrow B\right), A_{1} \vee A_{2} \rightarrow B\right\}$ or $\left\{\neg\left(A_{i}^{*} \rightarrow B\right), \neg\left(A_{1} \wedge\right.\right.$ $\left.A_{2}\right) \rightarrow B$. We analyze the first multiset and derivation; the analysis of the other can be developed along analogous lines. First of all it is straightforward to see that $\neg\left(A_{i} \rightarrow B\right), A_{1} \vee A_{2} \rightarrow B \vdash_{R} \perp$ (this also holds via Theorem 3.3). But it is evident that the following derivations hold as well: $\neg \neg\left(A_{i} \rightarrow B\right), A_{j} \rightarrow$ $B, \neg\left(A_{1} \vee A_{2} \rightarrow B\right) \vdash_{R} \perp$. Finally $\left\{\neg\left(A_{i} \rightarrow B\right), A_{j} \rightarrow B\right\}$ is completely and immediately less g-complex than $\neg\left(A_{1} \vee A_{2} \rightarrow B\right)$ according to Definition 2.8.

Lemma 4.2. For any composition of rules such that the formula $D$ is completely and immediately derivable from $\{A, M\},\{A, N\}$ (where one of these multisets might be empty), it holds that, not only $A, \wedge M \wedge \wedge N \vdash_{R} D$, but also $\bigwedge \neg(M) \wedge \bigwedge \neg(N), A \rightarrow D \vdash_{R} \perp$. Moreover the multiset $\{M, N\}$ is completely and immediately less g-complex than $A \rightarrow D$ according to the notion of g-complexity introduced in Definition 2.8 .

Proof. We prove this lemma by distinguishing the several forms that the derivation from $\{A, M\},\{A, N\}$ to $D$ might have (see Definition 3.7) and thus the several types these multisets might be. 
(1) $\mathrm{d}:=<\rightarrow E, \neg \neg I>$ or $\mathrm{d}:=\langle\neg \neg E, \rightarrow E>$ and it has one of these two forms,

$$
\frac{A \rightarrow B \quad A}{\frac{B}{\neg \neg B} \neg \neg I} \rightarrow E \quad \frac{A \rightarrow B \quad \frac{\neg \neg A}{A} \neg \neg E}{B} \rightarrow E
$$

Hence in these cases we only have one multiset $M$, which is either $\{A \rightarrow B, A\}$ or $\{A \rightarrow B, \neg \neg A\}$. In the first case $\neg \neg B$ can be relevantly derived, in the second case $B$ can be relevantly derived. Moreover in the first case it is straightforward to show that $\neg(A \rightarrow B), A \rightarrow \neg \neg B \vdash_{R} \perp$, while in the second case it is straightforward to show that $\neg(A \rightarrow B), \neg \neg A \rightarrow B \vdash_{R} \perp$. Finally $\{A \rightarrow B\}$ is completely and immediately less g-complex than $A \rightarrow \neg \neg B$ but also than $\neg \neg A \rightarrow B$ according to Definition 2.8.

(2) $\mathrm{d}:=<(\rightarrow E ; \rightarrow E), \wedge I>$ or $\mathrm{d}:=<(\rightarrow E ; \rightarrow E), \neg \vee>$ and it has one of these two forms,

$$
\frac{A, A \rightarrow B}{\frac{B}{B \wedge E} \quad \frac{A, A \rightarrow C}{C} \wedge I} \rightarrow E \quad \frac{A, A \rightarrow B^{*}}{\frac{B^{*}}{\neg(B \vee C} \quad \frac{A, A \rightarrow C^{*}}{C^{*}} \neg \vee} \rightarrow E
$$

In the derivation on the left the multisets are $\{A, A \rightarrow B\}$ and $\{A, A \rightarrow C\}$; in the derivation on the right the multisets are $\left\{A, A \rightarrow B^{*}\right\}$ and $\left\{A, A \rightarrow C^{*}\right\}$. We analyze the derivation and the multisets on the left; an analogous analysis can be drawn for the derivation and the multisets on the right. On the one hand, it is straightforward to see that from $A$ and $A \rightarrow B \wedge A \rightarrow C, B \wedge C$ can be relevantly derived (also via Theorem 3.3). On the other hand, it is evident that from $\neg(A \rightarrow B) \wedge \neg(A \rightarrow C)$ and $A \rightarrow(B \wedge C)$ a contradiction can be relevantly derived. Finally the multiset $\{A \rightarrow B, A \rightarrow C\}$ is completely and immediately less g-complex than $A \rightarrow B \wedge C$ according to Definition 2.8.

(3) $\mathrm{d}:=<(\rightarrow E ; \rightarrow E), \vee I>$ or $\mathrm{d}:=<(\rightarrow E ; \rightarrow E), \neg \wedge>$ and it has one of these two forms,

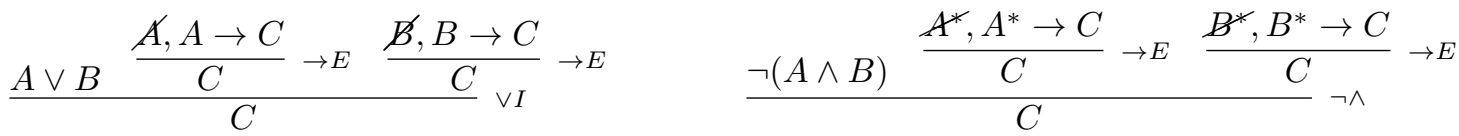

In the derivation on the left the multiset is $\{A \vee B, A \rightarrow C, B \rightarrow C\}$; in the derivation on the right the multiset is $\left\{\neg(A \wedge B), A^{*} \rightarrow C, B^{*} \rightarrow C\right\}$. We analyze the derivation and the multiset on the left; an analogous analysis can be drawn for the derivation and the multiset on the right. It is straightforward 
to show that from $A \vee B, A \rightarrow C \wedge B \rightarrow C, C$ can be relevantly derived (also via Theorem 3.3). But it also holds that from $\neg(A \rightarrow C) \wedge \neg(B \rightarrow C), A \vee B \rightarrow C$ a contradiction can be relevantly derived. Finally the multiset $\{A \rightarrow C, B \rightarrow C\}$ is completely and immediately less g-complex than $A \vee B \rightarrow C$ according to Definition 2.8.

Lemma 4.3. (Soundness) For any $C, M$ and $A \in \mathbb{P F}_{\rightarrow}$, if there is a complete and immediate formal explanation of $\boldsymbol{A}$ from $\boldsymbol{M}$ under the robust condition $\boldsymbol{C}$ according to Definition 3.8, then there is an immediate and complete formal grounding relation between $M$ and $A$ under the robust condition $C$ according to Definition 2.9.

Proof. We analyze each rule belonging to Figure 6 showing that, for any $C, M$ and $A$, if $\mathbf{C}, \mathbf{M}$ and $\mathbf{A}$ are linked by one of these rules, then $\mathrm{C}, M$ and $A$ satisfy the three conditions in Definition 2.9.

Let $M=\{D, E\}$ and suppose that

$$
\underline{\overline{\mathbf{D}} \mathbf{E}} \wedge \hat{\mathbf{D}} \wedge G
$$

So, by the form of the rule, $A \in \mathbb{A} \mathbb{C}(D \wedge E)$. Let $A=(D \wedge E)^{\prime} \in \mathbb{A} \mathbb{C}(D \wedge E)$. We must prove that

(a) $D \wedge E \vdash_{R}(D \wedge E)^{\prime}$

(b) $\neg D \wedge \neg E \vdash_{R} \neg(D \wedge E)^{\prime}$

(c) $\{D, E\}$ is completely and immediately less g-complex than $(D \wedge E)^{\prime}$ according to Definition 2.8.

By the way we have defined the objects belonging to the set $\mathbb{A} \mathbb{C}(D \wedge E)$ we have that: $D \wedge E \vdash(D \wedge E)^{\prime}$ which is condition (a), but also $\neg(D \wedge E) \vdash \neg(D \wedge E)^{\prime}$. From this by logic, we get condition (b). Since $(D \wedge E)^{\prime} \cong D \wedge E,\{D, E\}$ is completely and immediately less g-complex than $(D \wedge E)^{\prime}$ and condition (c) is satisfied.

Let $M=\{D, E\}$ and suppose that

$$
\frac{\mathbf{D} \mathbf{E}}{\overline{\mathbf{D} \vee \mathbf{E}} \vee G_{1}}
$$

So, by the form of the rule, $A \in \mathbb{A} \mathbb{C}(D \vee E)$. Let $A=(D \vee E)^{\prime} \in \mathbb{A} \mathbb{C}(D \vee E)$. We must prove that

(d) $D \wedge E \vdash_{R}(D \vee E)^{\prime}$

(e) $\neg D \wedge \neg E \vdash_{R} \neg(D \vee E)^{\prime}$

(f) $\{D, E\}$ is completely and immediately less g-complex than $(D \vee E)^{\prime}$ according to Definition 2.8. 
We establish (d)-(f) in the same way we have established the previous (a)-(c).

Let $M=\{D\}, C=E^{*}$ and suppose that (the analysis of the rule $\vee G_{3}$ is analogous):

$$
\frac{\left[\mathbf{E}^{*}\right] \quad \mathbf{D}}{\overline{\mathbf{D} \vee \mathbf{E}}} \vee G_{2}
$$

Let $A=(D \vee E)^{\prime} \in \mathbb{A} \mathbb{C}(D \vee E)$. We must prove that

(g) $D \vdash_{R}(D \vee E)^{\prime}$

(h) $\neg D, E^{*} \vdash_{R} \neg(D \vee E)^{\prime}$

(i) $\left\{D, E^{*}\right\}$ is completely and immediately less g-complex than $(D \vee E)^{\prime}$ according to Definition 2.8.

By the way we have defined the objects belonging to the set $\mathbb{A} C(D \vee E)$, we have that $D \vee E \vdash(D \vee E)^{\prime}$, but also $\neg(D \vee E) \vdash \neg(D \vee E)^{\prime}$. From the former and the fact that $D \vdash_{R} D \vee E$, by transitivity, we get condition (g); by the latter and the fact that $\neg D, E^{*} \vdash_{R} \neg(D \vee E)$, by transitivity, we get condition (h). Since $(D \vee E)^{\prime} \cong D \vee E$, then $\left\{D, E^{*}\right\}$ is completely and immediately less g-complex than $(D \vee E)^{\prime}$ and condition (i) is satisfied.

Let $M=\{D, E\}, A=\neg G$ and suppose that (a similar analysis can be developed for $\left.\neg G_{1}\right)$ :

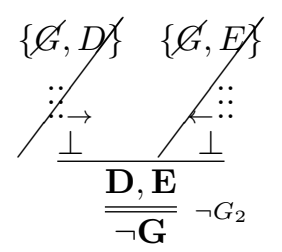

Let $\mathbf{A}=\neg G^{\prime} \in \mathbb{A} \mathbb{C}(\neg G)$. We must prove that, if $\{G, D\}$ and $\{G, E\}$ are two complementary completely and immediately contradictory multisets, then:

(j) $D \wedge E \vdash_{R} \neg G^{\prime}$

(k) $\neg D \wedge \neg E \vdash_{R} \neg \neg G^{\prime}$

(l) $\{D, E\}$ is completely and immediately less g-complex than $\neg G^{\prime}$ according to Definition 2.8.

Suppose that $\{G, D\}$ and $\{G, E\}$ are two complementary completely and immediately contradictory multisets. Then, by Lemma 4.1 , it follows that: (i) $G, D \vdash_{R} \perp$, (ii) $G, E \vdash_{R} \perp$, (iii) $\neg G, \neg D, \neg E \vdash_{R} \perp$, (iv) $\neg G$ is completely and immediately more g-complex than $\{D, E\}$. From (i) or (ii), by transitivity and either the axiom $A \wedge B \rightarrow A$ or the axiom $A \wedge B \rightarrow B$, it follows $G, D \wedge E \vdash_{R} \perp$. From this and the fact that $G^{\prime} \vdash_{R} G$, by transitivity, we have $G^{\prime}, D \wedge E \vdash_{R} \perp$. By Theorem 2.2, we get $D \wedge E \vdash_{R} \neg G^{\prime}$. From (iii) by transitivity and both 
the axioms $A \wedge B \rightarrow A$ and $A \wedge B \rightarrow B$, as well as the axiom $(A \rightarrow(A \rightarrow$ $B) \rightarrow(A \rightarrow B)$ ), it follows $\neg G, \neg D \wedge \neg E \vdash_{R} \perp$. From this and the fact that $G^{\prime} \vdash_{R} G$, by transitivity we have $\neg G^{\prime}, \neg D \wedge \neg E \vdash_{R} \perp$. By Theorem 2.2 we get $\neg D \wedge \neg E \vdash_{R} \neg \neg G^{\prime}$.

Let $M=\{D, E\}$ and suppose that:

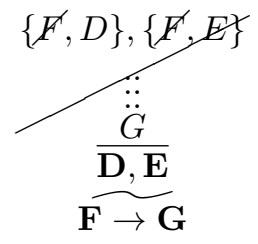

Let $\mathbf{A}=(F \rightarrow G)^{\prime} \in \mathbb{A} \mathbb{C}(F \rightarrow G)$. We must prove that, if from $\{F, D\}$ and $\{F, E\}, G$ can be completely and immediately derived, then:

(j) $D \wedge E \vdash_{R}(F \rightarrow G)^{\prime}$

(k) $\neg D \wedge \neg E \vdash_{R} \neg(F \rightarrow G)^{\prime}$

(l) $\{D, E\}$ is completely and immediately less g-complex than $(F \rightarrow G)^{\prime}$ according to Definition 2.8.

Suppose that from $\{F, D\}$ and $\{F, E\}, G$ can be completely and immediately derived. Then, by Lemma 4.2, it follows that: (i) $F, D \wedge E \vdash_{R} G$, (ii) $\neg D \wedge$ $\neg E, F \rightarrow G \vdash_{R} \perp$, (iii) $F \rightarrow G$ is completely and immediately more g-complex than $\{D, E\}$. From (i) and Theorem 2.2, it follows that $D \wedge E \vdash_{R} F \rightarrow G$. From this, the fact that $F \rightarrow G \vdash_{R}(F \rightarrow G)^{\prime}$ and transitivity we have $D \wedge E \vdash_{R}$ $(F \rightarrow G)^{\prime}$. By applying Theorem 2.2 to (ii), it follows $\neg D \wedge \neg E \vdash_{R} \neg(F \rightarrow G)$. From this and the fact that $\neg(F \rightarrow G) \vdash_{R}(\neg(F \rightarrow G))^{\prime}$, by transitivity we get the desired result.

Lemma 4.4. (Completeness) For any $C, M$ and $A \in \mathbb{P F}_{\rightarrow}$, if there is an immediate and complete formal grounding relation between $A$ and $M$ under the robust condition $C$ according to Definition 2.9, then there is a complete and immediate formal explanation of $A$ from $M$ under the robust condition $C$ according to Definition 3.8.

Proof. The general strategy to prove this Lemma is as follows. First, we carefully list those multisets $N$ and formulas $D$ such that $N$ is completely and immediately less g-complex than $D$ according to Definition 2.8. Then, amongst these $N$ and $D$, we identify the pairs that stand in positive, as well as in negative, derivability relation. Those $N$ and $D$ such that, not only $N$ is completely and immediately less g-complex than $D$, but also $N$ and $D$ stand in positive and negative derivability relation, constitute cases of complete and immediate formal grounding; we thus check whether these cases can also be counted as cases of formal explanation, i.e. whether those $N$ and $D$ can constitute the premisses and the conclusion, respectively, of one of our grounding rules. 
The proof is developed by induction on the g-complexity of $A$ (for the definition of the notion of g-complexity see Poggiolesi (2020a)). If the g-complexity of $A$ is 0 , and thus $A$ is either a literal (an atom or its negation) or an implication made of literals or the negation of an implication made of literals, then there is nothing that is completely and immediately less g-complex than $A$ and hence nothing that stands in a complete and immediate formal grounding relation with $A$. If the g-complexity of $A$ is $>0$, then we distinguish several cases (I) $A$ $\cong \neg \neg B$; (II) $A \cong(B \circ D$ ); (IIIa) $A \cong B \rightarrow \neg \neg C$, (IIIb) $A \cong \neg \neg B \rightarrow C$; (IVa) $A \cong B \circ C \rightarrow D$, (IIIb) $A \cong \neg(B \circ C) \rightarrow D ;$ (Va) $A \cong B \rightarrow C \circ D$, (Vb) $A \cong$ $B \rightarrow \neg(C \circ D)$. Note that cases (I) and (II) can be treated exactly as in the proof of the Completeness Lemma of Poggiolesi (2018); therefore we refer the reader to this paper. We will take into account cases (III)-(V).

In each of cases (III)-(V), the analysis of sub-case $a$ is analogous to the analysis of sub-case $b$, thus we will only deal with the former leaving the reader to deal with the latter.

(IIIa) If $A \cong B \rightarrow \neg \neg C$, then we have the following two sub-cases: (IIIa1) $A=E$ for some $E \in \mathbb{A} \mathbb{C}(B \rightarrow \neg \neg C)$ (IIIa2) $A=(\neg E)$ for some $E \in \mathbb{A} \mathbb{C}(B \rightarrow$ $\neg \neg C)$. The following two multi-sets $\{B \rightarrow C\}$ and $\{\neg(B \rightarrow C)\}$ are completely and immediately less g-complex than $A$, in each form - (IIIa1) or (IIIa2) - that $A$ might have. Adding to g-complexity positive and negative derivability, we have that:

- $\{B \rightarrow C\}$ stands in a relation of positive and negative derivability with $A$ $=E$ for some $E \in \mathbb{A} \mathbb{C}(B \rightarrow \neg \neg C)$

- $\{\neg(B \rightarrow C)\}$ stands in a relation of positive and negative derivability with $A=\neg E$ for some $E \in \mathbb{A} \mathbb{C}(B \rightarrow \neg \neg C)$,

Therefore, by Definition 2.9, $\{B \rightarrow C\}$ is the complete and immediate formal ground of $A$, when $A$ is of the form (IIIa1), while $\{\neg(B \rightarrow C)\}$ is the complete and immediate formal ground of $A$, when $A$ is of the form (IIIa2). On inspection, the rule $\rightarrow G_{1}$, with composition of rule $<\rightarrow E, \neg \neg I>$ ensures a complete and immediate formal explanation between $\{B \rightarrow \neg \neg C\}$ and $A$ when $A$ is of the form (IIIa1). On inspection, the rule $\neg G_{1}$, with composition of rules $\langle\rightarrow \neg \neg, \perp>$ ensures a complete and immediate formal explanation between $\{\neg(B \rightarrow C)\}$ and $A$ when $A$ is of the form (IIIa2). Thus, each case of formal grounding is also a case of formal explanation.

(IVa) If $A \cong B \circ C \rightarrow D$, then we have the following two sub-cases: (IVa1) $A$ $=E$ for some $E \in \mathbb{A} \mathbb{C}(B \circ C \rightarrow D)$ (IVa2) $A=(\neg E)$ for some $E \in \mathbb{A} \mathbb{C}(B \circ C \rightarrow$ $D)$. The following multi-sets $\{B \rightarrow C, B \rightarrow D\}$, or $\{\neg(B \rightarrow C), B \rightarrow D\}$, or $\{B \rightarrow C, \neg(B \rightarrow D)\}$, or $\{\neg(B \rightarrow C), \neg(B \rightarrow D)\}$ are all completely and immediately less g-complex than $A$, with $A$ being equal to $E$ or to $\neg E$.

Let us now make some further distinctions concerning the formula $A$ : 


$$
\begin{aligned}
& A=E \text { for some } E \in \mathbb{A} \mathbb{C}(B \wedge C \rightarrow D) \quad \text { IVa1^ } \\
& A=E \text { for some } E \in \mathbb{A} \mathbb{C}(B \vee C \rightarrow D) \quad \text { IVa1 } \vee \\
& A=\neg E \text { for some } E \in \mathbb{A} \mathbb{C}(B \wedge C \rightarrow D) \quad \text { IVa2^ } \\
& A=\neg E \text { for some } E \in \mathbb{A} \mathbb{C}(B \vee C \rightarrow D) \quad \text { IVa2 } \vee
\end{aligned}
$$

We now identify those multisets that are completely and immediately less g-complex than $A$ but also enjoy positive and negative derivability with $A$. We have:

$\odot$ The multiset $\{B \rightarrow D, C \rightarrow D\}$ stand in a relation of positive and negative

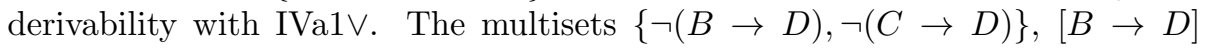
$\{\neg(C \rightarrow D)\},[C \rightarrow D]\{\neg(B \rightarrow D)\}$ stand in a relation of positive and negative derivability with IVa2 $\vee$.

$\ominus$ No multiset among those enumerated above stand in a relation of positive and negative derivability with IVa1^ and IVa2^.

From $\odot$, by Definition 2.9, it follows that $\{B \rightarrow D, C \rightarrow D\}$ is the complete and immediate formal ground of $A=E$ for some $E \in \mathbb{A C}(B \vee C \rightarrow D)$. On inspection, the rule $\rightarrow G$, with composition of rules $<(\rightarrow E ; \rightarrow E), \vee E>$, ensure a complete and immediate formal explanation relation between $\{B \rightarrow$ $D, C \rightarrow D\}$ and $A$, when $A=E$ for some $E \in \mathbb{A} \mathbb{C}(B \vee C \rightarrow D)$. Thus, this case of formal grounding is also a case of formal explanation.

From $\odot$, by Definition 2.9, it follows that $\{\neg(B \rightarrow D), \neg(C \rightarrow D)\}$ is the complete and immediate formal ground of $A=E$ for some $E \in \mathbb{A} C \neg(B \vee C \rightarrow$ $D)$. Also $\{\neg(C \rightarrow D)\}$ under the robust condition $[B \rightarrow D]$ is a complete and immediate formal ground of $A=E$ for some $E \in \mathbb{A} C \neg(B \vee C \rightarrow D)$, but also $\{\neg(B \rightarrow D)\}$ under the robust condition $[C \rightarrow D]$ is the complete and formal ground of $A=E$ for some $E \in \mathbb{A} C \neg(B \vee C \rightarrow D)$. On inspection, the rules $\neg G_{1}$ and $\neg G_{2}$, with composition of rules $\left\langle\vee_{i} \rightarrow, \perp\right\rangle$, ensures a complete and immediate formal explanation relation between $\{\neg(B \rightarrow D), \neg(C \rightarrow D)\}$, $[B \rightarrow D]\{\neg(C \rightarrow D)\}$, but also $[C \rightarrow D]\{\neg(B \rightarrow D)\}$, and $A$, when $A=\neg E$ for some $E \in \mathbb{A} \mathbb{C}(B \vee C \rightarrow D)$. Thus, each of these cases of formal grounding is also a case of formal explanation.

From $\ominus$ nothing formally grounds $A=E$ for some $E \in \mathbb{A} \mathbb{C}(B \wedge C \rightarrow D)$, nor $A=\neg E$ for some $E \in \mathbb{A} \mathbb{C}(B \wedge C \rightarrow D)$, since no mutliset that is less g-complex than $A$ in either of these two forms enjoys positive and negative derivability with $A$.

(Va) If $A \cong B \rightarrow C \circ D$, then we have the following two sub-cases: (Va1) $A=$ $E$ for some $E \in \mathbb{A} \mathbb{C}(B \rightarrow C \circ D)($ Va2) $A=(\neg E)$ for some $E \in \mathbb{A} C(B \rightarrow C \circ D)$. The following multi-sets $\{B \rightarrow C, B \rightarrow D\}$, or $\{\neg(B \rightarrow C), B \rightarrow D\}$, or $\{B \rightarrow C, \neg(B \rightarrow D)\}$, or $\{\neg(B \rightarrow C), \neg(B \rightarrow D)\}$ are all completely and 
immediately less g-complex than $A$, with $A$ being equal to $E$ or to $\neg E$.

Let us now make some further distinctions concerning the formula $A$ :

$$
\begin{aligned}
& A=E \text { for some } E \in \mathbb{A} \mathbb{C}(B \rightarrow C \wedge D) \quad \text { Va1^ } \\
& A=E \text { for some } E \in \mathbb{A} \mathbb{C}(B \rightarrow C \vee D) \quad \text { Va1 } \vee \\
& A=\neg E \text { for some } E \in \mathbb{A} \mathbb{C}(B \rightarrow C \wedge D) \quad \text { Va2^ } \\
& A=\neg E \text { for some } E \in \mathbb{A} \mathbb{C}(B \rightarrow C \vee D) \quad \text { Va2 } \vee
\end{aligned}
$$

We now identify those multisets that are completely and immediately less g-complex than $A$ but also enjoy positive and negative derivability with $A$. We have:

$\odot$ The multiset $\{B \rightarrow C, B \rightarrow D\}$ stand in a relation of positive and negative derivability with Va1^. The multisets $\{\neg(B \rightarrow C), \neg(B \rightarrow D)\},[B \rightarrow C]$ $\{\neg(B \rightarrow D)\},[B \rightarrow D]\{\neg(B \rightarrow C)\}$ stand in a relation of positive and negative derivability with $\operatorname{Va} 2 \wedge$.

$\ominus$ No multiset among those enumerated above stand in a relation of positive and negative derivability with Va1 $\vee$ and Va2 $\vee$.

From $\odot$, by Definition 2.9, it follows that $\{B \rightarrow C, B \rightarrow D\}$ is the complete and immediate formal ground of $A=E$ for some $E \in \mathbb{A C}(B \rightarrow C \wedge D)$. On inspection, the rule $\rightarrow G$, with composition of rules $\langle(\rightarrow E ; \rightarrow E), \wedge I>$, ensures a complete and immediate formal explanation relation between $\{B \rightarrow$ $D, C \rightarrow D\}$ and $A$, when $A=E$ for some $E \in \mathbb{A} \mathbb{C}(B \rightarrow C \wedge D)$. Thus, this case of formal grounding is also a case of formal explanation.

From $\odot$, by Definition 2.9, it follows that $\{\neg(B \rightarrow C), \neg(B \rightarrow D)\}$ is the complete and immediate formal ground of $A=E$ for some $E \in \mathbb{A} \neg \neg(B \rightarrow$ $C \wedge D)$. Also $\{\neg(B \rightarrow D)\}$ under the robust condition $[B \rightarrow C]$ is a complete and immediate formal ground of $A=E$ for some $E \in \mathbb{A} C \neg(B \rightarrow C \wedge D)$, but also $\{\neg(B \rightarrow C)\}$ under the robust condition $[B \rightarrow D]$ is the complete and

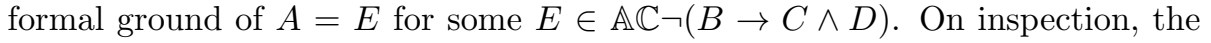
rules $\neg G_{1}$ and $\neg G_{2}$, with composition of rules $\left\langle\rightarrow \wedge_{i}, \perp\right\rangle$, ensures a complete and immediate formal explanation relation between $\{\neg(B \rightarrow C), \neg(B \rightarrow D)\}$, $[B \rightarrow C]\{\neg(B \rightarrow D)\}$, but also $[B \rightarrow D]\{\neg(B \rightarrow C)\}$, and $A$, when $A=\neg E$ for some $E \in \mathbb{A} \mathbb{C}(B \vee C \rightarrow D)$. Thus, each of these cases of formal grounding is also a case of formal explanation.

From $\ominus$ nothing formally grounds $A=E$ for some $E \in \mathbb{A} \mathbb{C}(B \rightarrow C \vee D)$, nor $A=\neg E$ for some $E \in \mathbb{A} \mathbb{C}(B \rightarrow C \vee D)$, since no multiset that is less g-complex than $A$ in either of these two forms enjoys positive and negative derivability with $A$. 
Theorem 4.5. (Soundness and Completeness) The relation of complete and immediate formal explanation of Definition 3.8 is sound and complete with respect to the notion of complete and immediate formal grounding of Definition 2.9 .

Proof. It follows from Lemmas 4.3 and 4.4 .

\section{Conservativity and grounding deduction the- orem}

We use this section to show two final results. The first result concerns the relation between the relevant deduction calculus $\mathcal{R D}$ and our calculus $\mathcal{R G D}$ : we will show that the latter calculus conservatively extends the former. The second result concerns the two symbols $\mid \sim$ and $\triangleright$. As the standard deduction theorem shows a deep link between derivability and implication, in the same way we will show a deep link between formal explanation $\mid \sim$ and the connective because $\triangleright$, via a grounding deduction theorem.

Theorem 5.1. For any formula $A \in \mathcal{L}^{r}$, we have that:

$$
\vdash_{\mathcal{R D}} A \text { if, and only if, } \mid \simeq_{R G D} A
$$

Proof. From left to right it is straightforward, since all the rules of the relevant deduction calculus are also rules of the $\mathcal{R G D}$ calculus. Let us then analyze the direction from right to left. We distinguish cases according to the last rule used to derive $A$, taking into account the fact that all assumptions need to be discharged and that $A$ cannot contain the symbol $\triangleright$ (because $A \in \mathcal{L}^{r}$ ). Then either $A$ has been obtained by the rule $\rightarrow I$, or by one of the rules $\triangleright E_{1}, \triangleright E_{2}$, where the premise of these rules is not an assumption. Note that the rule $\triangleright E_{3}$ cannot have been used to derive $A$ for the following reason. Since the premise of this rule, which is a formula of the form $C, M \triangleright B$, cannot be an assumption, then $C, M \triangleright B$ has been obtained by a formal explanation followed by the rule $\triangleright I$. But if this is the case then, by Lemma 4.3, we know that the multiset $C \cup M$ is not inconsistent and that it is not more g-complex than $A$. Thus in this case the rule $\triangleright E_{3}$ cannot be applied.

Suppose that the last rule used to derive $A$ is $\rightarrow I$. Then by induction on the length of the derivation, the case is trivial. Suppose then that the last rule used to obtain $A$ is the rule $\triangleright E_{1}$, then, $A$ is of the form $\bigwedge M \rightarrow B$ and it has been derived by $C, M \triangleright B$. Since $C, M \triangleright B$ cannot be an assumption, then $C, M \triangleright B$ has been obtained by a formal explanation via the rule $\triangleright I$. Then by Lemma 4.3, we know that we have $\bigwedge M \vdash_{R} B$. So, by soundness between the relevant Hilbert system $\mathbf{R}$ and the relevant deduction system $\mathcal{R D}$, see Theorem 3.3, we also have $\bigwedge M_{\alpha} \vdash_{\mathcal{R D}} B_{\alpha}$. From this and the rule $\rightarrow I$, we get $\vdash_{\mathcal{R D}} \wedge M \rightarrow B$.

If the last rule used to obtain $A$ is the rule $\triangleright E_{2}$, then $A$ is of the form $C \wedge \wedge \neg(M) \rightarrow \neg B$ and it has been derived by $C, M \triangleright B$. Since $C, M \triangleright B$ cannot be an assumption, then it has been obtained by a formal explanation via the 
rule $\triangleright I$. Then, by Lemma 4.3, we know that we have $C, \bigwedge \neg(M) \vdash_{R} \neg B$. So, by soundness between the relevant Hilbert system $\mathbf{R}$ and the relevant deduction calculus $\mathcal{R D}$, see Theorem 3.3, we also have $C \wedge \bigwedge \neg(M)_{\alpha} \vdash_{\mathcal{R D}} \neg B_{\alpha}$. From this and the rule $\rightarrow I$, we get $\vdash_{\mathcal{R D}} C \wedge \wedge \neg(M) \rightarrow \neg B$.

Corollary 5.2. The calculus $\mathcal{R G D}$ is consistent, namely it is not the case that $\mid \simeq_{R G D} \perp$.

Proof. It follows straightforwardly from the previous theorem.

Theorem 5.3. For any multiset $M$ and formula $A \in \mathbb{P F}_{\rightarrow}$, we have that:

$$
M \mid \sim_{R} A \text { if, and only if, } \mid \simeq_{R G D} M \triangleright A
$$

Proof. From left to right, we simply use the rule $\triangleright I$ to get the desired result. Let us then analyze the side from right to left. If $\mid \simeq_{R G D} M \triangleright A$, then by the rule $\triangleright E_{1}$, we get $\vdash_{\mathcal{R} \mathcal{D}} \wedge M \rightarrow A$. From this, by Theorem 3.3 and Theorem 2.2, we have (i) $\bigwedge M \vdash_{R} A$.

If $\mid \simeq_{R G D} M \triangleright A$, then by the rule $\triangleright E_{2}$, we get $\vdash_{\mathcal{R D}} C \wedge \wedge \neg(M) \rightarrow \neg A$. From this, by Theorem 3.3 and Theorem 2.2, we have (ii) $C, \wedge \neg(M) \vdash_{R} \neg A$.

By Corollary $5.2, \mathcal{R G D}$ is a consistent calculus. Since the rule $\triangleright E_{3}$ can be read as saying that if not $\mid \bigwedge_{R G D} \perp$, then not $\mid \bigwedge_{R G D} C, M \triangleright A$, where $\{C, M\}$ is inconsistent or more g-complex than $A$, from this and the fact that $\mid \simeq_{R G D} C, M \triangleright A$, we have that (iii) $\{C, M\}$ is a consistent multiset and is less g-complex than $A$.

From (i)-(iii) and Lemma 4.4, we have that $M \mid \sim_{R} A$.

\section{Conclusion}

In this paper, by relying on previous results concerning the grounding principles for (relevant) implication, we have presented a calculus containing two grounding rules for implication that allow us to prove such principles. The calculus we have presented is built upon the relevant natural deduction calculus $\mathcal{R} \mathcal{D}$, it conservatively extends that calculus and it enjoys the deduction theorem at the grounding level. Hence, this work not only represents a solid logic counterpart of the results obtained in Poggiolesi (2020a), but also it makes the first steps towards showing the interest of developing a grounding framework for logics different from classical logic. We believe that this undeveloped area of research might turn out to be thriving.

\section{References}

Anderson, A. R. and Belnap, N. (1975). Entailement. The logic of relevance and necessity. Princeton University Press. 
Correia, F. (2010). Grounding and truth-functions. Logique et Analyse, 53(211):251-79.

Correia, F. (2014). Logical grounds. Review of Symbolic Logic, 7(1):31-59.

Dunn, M. (1970). Algebraic completeness for r-mingle and its extensions. Journal of Symbolic Logic, 25:1-13.

Dunn, M. and Restall, G. (2002). Relevance logic. In Gabbay, D. and Guenthner, F., editors, Handbook of Philosophical Logic, pages 291-309. Kluwer Academic Publisher.

Fine, K. (1974). Models for entailment. Journal of Philosophical Logic, 3:347372 .

Fine, K. (2012a). Guide to ground. In Correia, F. and Schnieder, B., editors, Metaphysical grounding, pages 37-80. Cambridge University Press, Cambridge.

Fine, K. (2012b). The pure logic of ground. Review of Symbolic Logic, 25(1):125.

Korbmacher, J. (2017). Axiomatic theories of partial ground I. The base theory. Journal of Philosophical Logic, 47:161-191.

Mares, E. (2014). Relevance logic. In Zalta, E. N., editor, The Stanford Encyclopedia of Philosophy.

McSweeney, M. (2020). Grounding logically complex facts. In Raven, M., editor, Routledge Handbook of metaphysical grounding, pages 228-242. Routledge.

Poggiolesi, F. (2016a). A critical overview of the most recent logics of grounding. In Boccuni, F. and Sereni, A., editors, Objectivity, Realism and Proof. Boston Studies in the Philosophy and History of Science.

Poggiolesi, F. (2016b). On defining the notion of complete and immediate formal grounding. Synthese, 193:3147-3167.

Poggiolesi, F. (2018). On constructing a logic for the notion of complete and immediate formal grounding. Synthese, 195:1231-1254.

Poggiolesi, F. (2020a). Grounding principles for (relevant) implication. Synthese, pages $1-28$.

Poggiolesi, F. (2020b). A proof-based framework for several types of grounding. HAL, hal-02498116, 3:1-34.

Routley, R. and Meyer, R. K. (1973). Semantics of entailment. In Leblanc, H., editor, Truth Syntax and Modality. North Holland.

Schnieder, B. (2011). A logic for 'Because'. The Review of Symbolic Logic, $4(03): 445-465$. 
Smithson, R. (2019). Metaphysical and conceptual grounding. Erkenntnis, forthcoming:1-25.

Urquhart, A. (1972). Semantics for relevant logics. Journal of Symbolic Logic, $37: 159-169$. 